\title{
Synthesis of antimalarial amide analogues based on the plant serrulatane diterpenoid 3,7,8-trihydroxyserrulat-14-en-19-oic acid
}

\author{
Rohitesh Kumar, Sandra Duffy, Vicky M. Avery, and Rohan A. Davis* \\ Griffith Institute for Drug Discovery, Griffith University, Brisbane, QLD 4111
}

\section{ARTICLE INFO}

Article history:

Received

Received in revised form

Accepted

Available online

Keywords:

Natural product scaffold

Eremophila microtheca

Serrulatane

Amides

Anti-plasmodial

\section{ABSTRACT}

A plant-derived natural product scaffold, 3,7,8-trihydroxyserrulat-14-en-19-oic acid (1) was isolated in high yield from the aerial parts of the endemic Australian desert plant Eremophila microtheca. This scaffold (1) was subsequently used in the generation of a series of new amide analogues via a one-pot mixed anhydride amidation using pivaloyl chloride. The structures of all analogues were characterized using MS, NMR, and UV data. The natural products (1-3) and all amide analogues (6-15) together with several pivaloylated derivatives of 3,7,8trihydroxyserrulat-14-en-19-oic acid (16-18) were evaluated for their antimalarial activity against 3D7 (chloroquine sensitive) and Dd2 (chloroquine resistant) Plasmodium falciparum strains, and preliminary cytotoxicity data were also acquired using the human embryonic kidney cell line HEK293. The natural product scaffold (1) did not display any antimalarial activity at 10 $\mu \mathrm{M}$. Replacing the carboxylic acid of $\mathbf{1}$ with various amides resulted in moderate activity against the $P$. falciparum $3 \mathrm{D} 7$ strain with $\mathrm{IC}_{50}$ values ranging from 1.25 to $5.65 \mu \mathrm{M}$.

2016 Elsevier Ltd. All rights reserved.

\section{Introduction}

Natural products (NPs) continue to have a significant impact in the field of drug discovery ${ }^{1,2}$ with an increasing number of Food \& Drug Administration (FDA) approved drugs either NPs or NP derivatives. ${ }^{2-4}$ Whilst de novo multi-step synthesis of bioactive NPs coupled with medicinal chemistry and analogue synthesis is one important strategy for drug development, ${ }^{3,5-7}$ the chemical modification of isolated NP scaffolds for the generation of diverse and focused libraries is another method. ${ }^{8}$ Fredericamycin A, ${ }^{9,10}$ manzamine $A,{ }^{11,12}$ and lipopeptide FR901379 13,14 are examples of such an approach. ${ }^{8}$

Our approach to the generation of chemically diverse and unique screening libraries for drug discovery and chemical biology purposes is based on the use of isolated NPs from Australian biota such as plant (e.g. muurolane library), ${ }^{15}$ mushrooms (e.g. tetrahydroanthraquinone library), ${ }^{16}$ and endophytic fungi (e.g. phenylacetic acid library). ${ }^{17}$ As part of our on-going research and interest in this field, the abundant NP 3,7,8-trihydroxyserrulat-14-ene-19-oic acid (1) isolated from the endemic Australian desert plant, Eremophila microtheca was identified as an attractive scaffold for structural modification and library generation. This NP scaffold was identified during a previous chemical investigation of the aerial parts of this plant, which also yielded the related serrulatane diterpenoids, 3acetoxy-7,8-dihydroxyserrulat-14-en-19-oic acid (2) and 3,19diacetoxy-8-hydroxyserrulat-14-ene (3) together with the known compounds verbascoside (4) and jaceosidin (5) (Fig. 1). ${ }^{18}$ The serrulatane skeleton has been shown to display a wide range of biological properties including antibacterial, ${ }^{18,19}$ antituberculosis, ${ }^{20}$ and antifouling ${ }^{21}$ activities. Due to its high abundance, low Mw (348 Da), multiple stereogenic centres ( $\mathrm{n}=$ 4) and potential chemical handles (i.e. the carboxylic acid,

secondary hydroxy and phenolic groups) compound $\mathbf{1}$ was deemed to be a particularly attractive NP scaffold for the generation of amide analogues.

The amide functionality is widespread in naturally occurring and synthetic compounds and has significant importance to pharmaceutical research. ${ }^{22}$ Amides are present in $25 \%$ of available drugs and amidation reactions are among the most commonly used reactions in medicinal chemistry. ${ }^{22-26}$ Herein, the re-isolation of the E. microtheca metabolite, 3,7,8trihydroxyserrulat-14-en-19-oic acid (1) and the semi-synthesis of amide analogues based on $\mathbf{1}$ are reported. The structural elucidation together with the antimalarial evaluation of the derivatives are also reported.

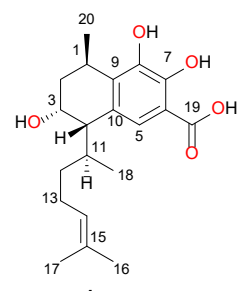

1

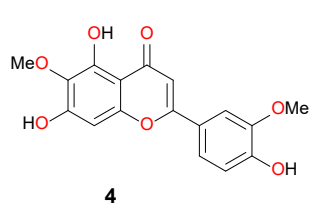

Figure 1. Structures of secondary metabolites isolated from aerial parts of E. microtheca. 


\section{Results and discussion}

The $\mathrm{CH}_{2} \mathrm{Cl}_{2}$ extract of the air-dried and ground aerial parts of E. microtheca was chromatographed on preparative C18 HPLC followed by semi-preparative C18 HPLC to afford the desired NP scaffold, compound 1 in high yields $(2.1 \%$ dry wt). The structure of 1 was verified after NMR, $[\alpha]_{D}$ and MS data analysis and comparison with literature values. ${ }^{18}$ Attempts to form serrulatane-based amides under standard conditions using coupling agents such as EDCI, ${ }^{27} \mathrm{~T} 3 \mathrm{P},{ }^{24,25,27}$, oxalyl chloride ${ }^{28}$ and HBTU $^{24,25,27}$ were unproductive.

Finally, a one-pot process where a mixed anhydride is prepared in situ as an intermediate and subsequently reacted with the desired amines proved successful. This was achieved by activation of the carboxylic acid to form a mixed pivalic anhydride ${ }^{25}$ by addition of pivaloyl chloride to compound $\mathbf{1}$ in pyridine followed by the addition of selected amine(s) (Scheme $1)$.

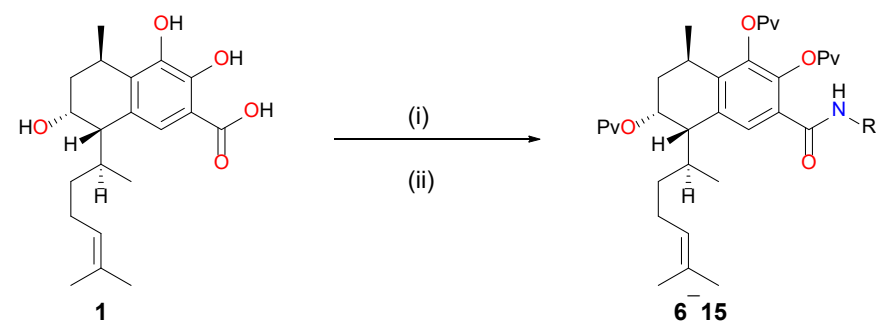

Scheme 1: Two-step coupling procedure via pivalic anhydride: (i) $\mathrm{PvCl}$, pyridine, $0^{\circ} \mathrm{C}, 2 \mathrm{~h}$. (ii) amine, rt, $16 \mathrm{~h}$.

A total of 10 amides (6-15) (Fig. 2) were semi-synthesized via this procedure and were purified by $\mathrm{C}_{18}$ HPLC with the yields ranging from $2-18 \%$. During the purification process some starting material was recovered along with three byproducts of the coupling reaction with different degrees of pivaloylation (1618) (Fig. 3) with the yields ranging from $7-16 \%$.

The structures of all the amide analogues (6-15) and the pivaloylated serrulatanes (16-18) were determined following 1D $\left({ }^{1} \mathrm{H}\right.$ and ${ }^{13} \mathrm{C}$ ) and 2D NMR (COSY, HSQC, HMBC, and ROESY) and HRESIMS data analysis. For example, the (+)-HRMS spectrum of 4.6 revealed an ion at $\mathrm{m} / \mathrm{z} 726.4335[\mathrm{M}+\mathrm{Na}]+$ that corresponded to the molecular formula, $\mathrm{C}_{43} \mathrm{H}_{61} \mathrm{NO}_{7}$, of the desired product ( $\mathrm{m} / \mathrm{z}$ calcd for $\mathrm{C}_{43} \mathrm{H}_{61} \mathrm{NO}_{7} \mathrm{Na}, 726.4340$ ).
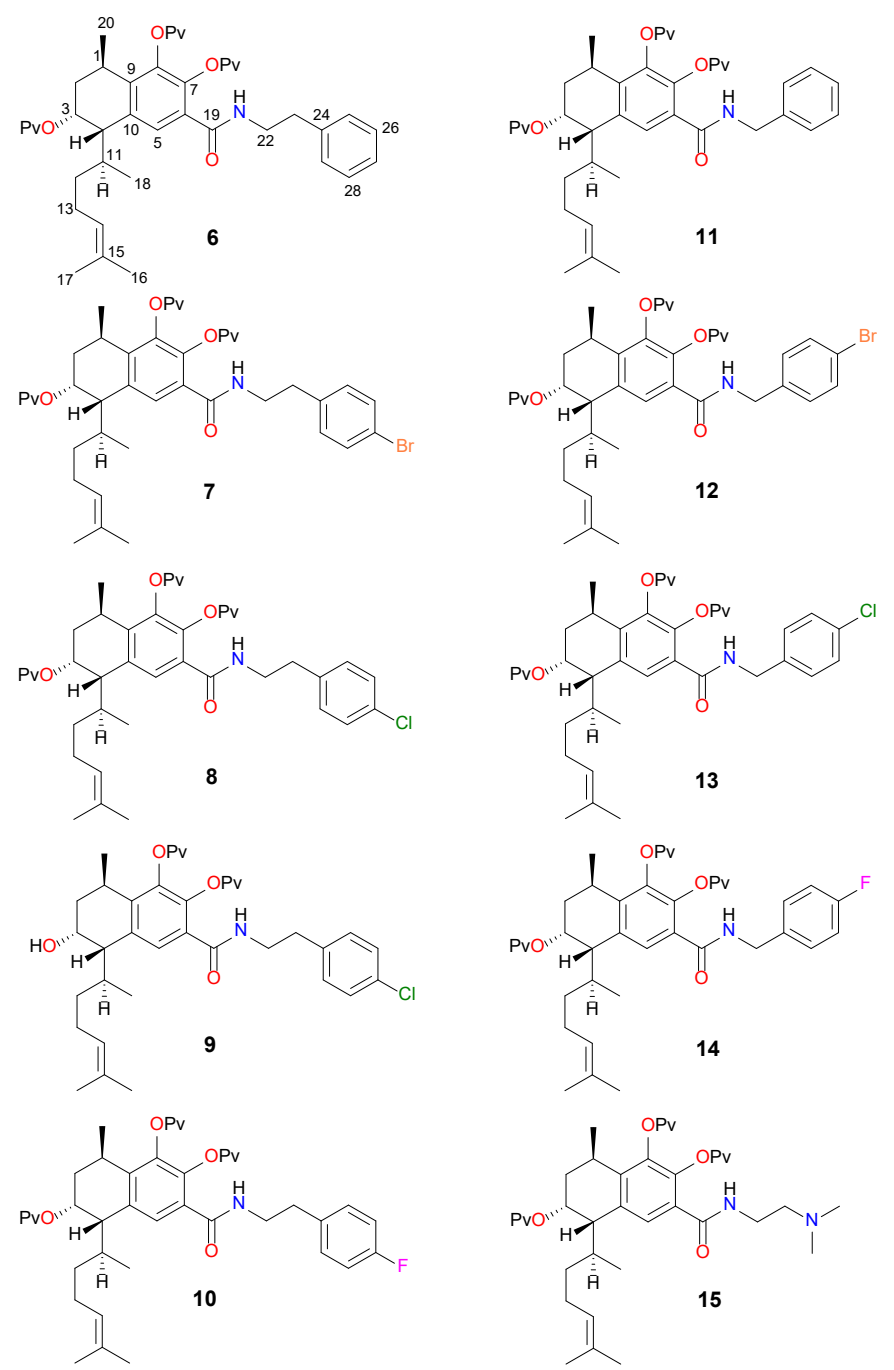

Figure 2. Chemical structures of serrulatane amides 6-15.<smiles>CCCCOc1c(C(=O)O)cc2c(c1OCCC)C(C)C[C@H](O)[C@H]2[C@H](C)CCC=C(C)C</smiles>

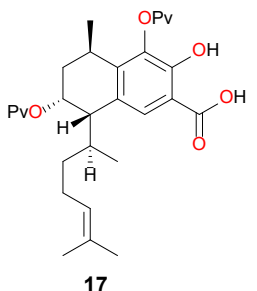<smiles>CCCCOc1c(C(=O)O)cc2c(c1OCCC)C(C)C[C@@H](O)[C@]2(C)C(C)CCC=C(C)C</smiles>

Figure 3. Chemical structures of pivaloylated serrulatanes 16-18.

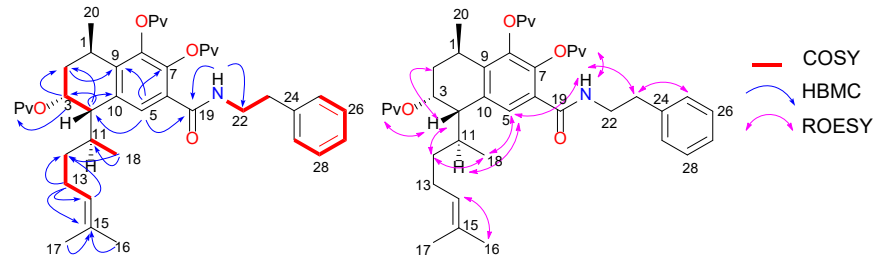

Figure 4. Selected COSY, HMBC and ROESY correlations for 6.

The ${ }^{1} \mathrm{H}$ NMR spectrum of compound 6 indicated the presence of one amide proton $\left(\delta_{\mathrm{H}} 8.43\right)$, six aromatic protons $\left(\delta_{\mathrm{H}} 7.02\right.$, $7.19,2 \times 7.23,2 \times 7.28)$, one olefinic proton $\left(\delta_{\mathrm{H}} 5.12\right)$, four methine $\left(\delta_{\mathrm{H}} 2.08,2.95,3.04,5.20\right)$, five methylene $\left(\delta_{\mathrm{H}} 1.61 / 2.10\right.$, $1.28 / 1.40,1.95 / 2.02,2.78,3.34 / 3.39)$, four methyl $\left(\delta_{\mathrm{H}} 0.53,1.20\right.$, $1.57,1.65)$ and three pivaloyl protons $\left(\delta_{\mathrm{H}} 1.14,1.24,1.31\right)$. The ${ }^{13} \mathrm{C}$ NMR and the HSQC spectra of $\mathbf{4 . 6}$ indicated a total of 43 carbons (Table 1) including four methyls $\left(\delta_{\mathrm{C}} 17.5,18.4,22.8\right.$, $25.5)$, five methylenes $\left(\delta_{\mathrm{C}} 25.8,31.0,34.9,37.7,40.7\right)$, four methines $\left(\delta_{\mathrm{C}} 22.8,31.0,45.7,68.8\right)$, two olefinic carbons $\left(\delta_{\mathrm{C}}\right.$ $124.2,130.9), 12$ aromatic carbons $\left(\delta_{\mathrm{C}} 126.00,126.04,2 \times 128.3\right.$, 
$128.5,2 \times 128.7,134.5,136.6,138.9,139.5,140.8)$, one carbonyl carbon $\left(\delta_{\mathrm{C}} 165.0\right)$ and 15 carbon signals belonging to three pivaloyl units. The exchangeable triplet at $\delta_{\mathrm{H}} 8.43(J=5.6 \mathrm{~Hz})$ showed HMBC correlations to C-19 (carbonyl) and C-22 and also showed a strong COSY correlation to $\mathrm{H}-22$, thus confirming the amide linkage of compound 6 (Fig. 4). The remaining NMR signals were assigned following comparison of the chemical shifts with the previously report natural product, compound $\mathbf{1}^{18}$ (Table 1), and further analysis of the 2D NMR data of 6.

Owing to our interest in identifying potential antimalarial compounds from natural sources ${ }^{15,17,29,30}$ the NPs isolated from $E$. microtheca and the analogues generated were screened for their anti-plasmodial activities. To date there have been no reports of antimalarial activity associated with the serrulatane structural class.

All compounds (1-18) were provisionally tested for antiplasmodial activity against Plasmodium falciparum 3D7 at four decreasing doses. The parent molecule (1) plus a related serrulatane (2), demonstrated no activity at $10 \mu \mathrm{M}$. The serrulatane (3), amide analogues (6-15) together with the pivaloylated derivatives (16-18) of 1 were all tested further for their antimalarial activity against both $P$. falciparum 3D7 (chloroquine sensitive) and $\mathrm{Dd} 2$ (multidrug resistant), and preliminary cytotoxicity data acquired using the human embryonic kidney cell line HEK293 (Table 2). Of the three NPs isolated from $E$. microtheca only compound $\mathbf{3}$ exhibited $\mathrm{IC}_{50}$ values of 6.89 and $8.76 \mu \mathrm{M}$ against $3 \mathrm{D} 7$ and $\mathrm{Dd} 2$ strains This data suggested that the secondary alcohol at C-3 does not play an important role in the antimalarial properties of these structurally similar serrulatanes. However, replacement of the carboxylic acid with a methyl acetate at C-6 improved the activity. The pivaloylated analogues, 16-18 displayed weak antimalarial activity with $\mathrm{IC}_{50}$ values ranging from $8.44-13.62 \mu \mathrm{M}$ against the 3D7 strain further supporting the hypothesis that the carboxylic acid is not required for antimalarial activity. 
Table 1: NMR data of compounds 1 and 6 in DMSO- $d_{6}$

\begin{tabular}{|c|c|c|c|c|}
\hline \multirow[t]{2}{*}{ Position } & \multicolumn{2}{|c|}{ Compound $\mathbf{1}^{a}$} & \multicolumn{2}{|c|}{ Compound $\mathbf{6}^{b}$} \\
\hline & ${ }^{1} \mathrm{H}$ (mult., $J$ in $\mathrm{Hz}$ ) & ${ }^{13} \mathrm{C}$ & ${ }^{1} \mathrm{H}$ (mult., $J$ in $\mathrm{Hz}$ ) & ${ }^{13} \mathrm{C}$ \\
\hline 1 & $3.19, \mathrm{br} \mathrm{dq}(7.2,7.2)$ & 28.5 & 2.95, br m & 22.8 \\
\hline $2 \alpha$ & 1.87, ddd $(12.5,12.0,7.2)$ & 34.6 & $2.10^{d}$ & 31.0 \\
\hline $2 \beta$ & $1.45, \mathrm{~m}$ & & $1.61, \mathrm{~m}$ & \\
\hline 3 & 4.05 , br dd $(12.5,4.2)$. & 64.9 & $5.20, \mathrm{dt}(11.0,4.1)$ & 68.8 \\
\hline $3-\mathrm{OH}$ & 4.62, br s & & & \\
\hline $3-\mathrm{OPv}$ & & & $1.14, \mathrm{~s}$ & $176.8, \mathrm{C} ; 38.3, \mathrm{C} ; 27.76, \mathrm{CH}_{3}$ \\
\hline 4 & 2.69, br d (4.2) & 48.2 & 3.04, br d (4.5) & 45.7 \\
\hline 5 & $6.94, \mathrm{~s}$ & 120.0 & $7.02, \mathrm{~s}$ & 126.00 \\
\hline 6 & & 110.1 & & 128.5 \\
\hline 7 & & 147.4 & & 138.9 \\
\hline 7-OH & $c$ & & & \\
\hline 7-OPv & & & $1.24, \mathrm{~s}$ & $174.8, \mathrm{C} ; 38.5, \mathrm{C} ; 26.84, \mathrm{CH}_{3}$ \\
\hline 8 & & & & 140.8 \\
\hline $8-\mathrm{OH}$ & 8.60 , br s & 142.3 & & \\
\hline $8-\mathrm{OPv}$ & & & $1.31, \mathrm{~s}$ & $174.8, \mathrm{C} ; 38.6, \mathrm{C} ; 26.9, \mathrm{CH}_{3}$ \\
\hline 9 & & 135.3 & & 136.6 \\
\hline 10 & & 128.3 & & 134.5 \\
\hline 11 & 2.10, br dq $(13.8,6.6)$ & 29.9 & $2.08^{d}$ & 31.0 \\
\hline $12 \mathrm{a}$ & $1.47, \mathrm{~m}$ & 38.5 & $1.40, \mathrm{~m}$ & 37.7 \\
\hline $12 b$ & 1.25, br ddt $(13.8,7.8,7.8)$ & & $1.28^{e}$ & \\
\hline 13 & 1.97, ddd $(7.8,7.2,7.2)$ & 25.7 & $\begin{array}{l}2.02, \mathrm{~m} \\
1.95, \mathrm{ddd}(7.9,7.8,7,8)\end{array}$ & 25.8 \\
\hline 14 & $5.13, \mathrm{t}(7.2)$ & 124.8 & $5.12, \mathrm{t}(7.0)$ & 124.2 \\
\hline 15 & & 130.2 & & 130.9 \\
\hline 16 & 1.64 , br s & 25.5 & $1.65(\mathrm{~s})$ & 25.5 \\
\hline 17 & 1.57, br s & 17.5 & $1.57(\mathrm{~s})$ & 17.5 \\
\hline 18 & $0.36, \mathrm{~d}(6.6)$ & 18.8 & $0.53, \mathrm{~d}(6.8)$ & 18.4 \\
\hline 19 & & 172.4 & & 165.0 \\
\hline 20 & $1.17, \mathrm{~d}(7.2)$ & 21.9 & $1.20^{e}$ & 22.8 \\
\hline $21-\mathrm{NH}$ & & & $8.43, \mathrm{t}(5.6)$ & \\
\hline $22 \mathrm{a}$ & & & $3.39, \mathrm{~m}$ & 40.7 \\
\hline $22 b$ & & & $3.34, \mathrm{~m}$ & \\
\hline 23 & & & $2.78, \mathrm{~m}$ & 34.9 \\
\hline 24 & & & & 139.5 \\
\hline 25 & & & $7.23, \mathrm{~d}(7.4)$ & 128.7 \\
\hline 26 & & & $7.28, \mathrm{dd}(7.4,7.4)$ & 128.3 \\
\hline 27 & & & 7.19, dd $(7.4,7.4)$ & 126.04 \\
\hline 28 & & & 7.28, dd $(7.4,7.4)$ & 128.3 \\
\hline 29 & & & $7.23, \mathrm{~d}(7.4)$ & 128.7 \\
\hline
\end{tabular}

${ }^{a}$ Recorded at $600 \mathrm{MHz}\left({ }^{1} \mathrm{H} \mathrm{NMR}\right)$ and $150 \mathrm{MHz}\left({ }^{13} \mathrm{C}\right.$ NMR $)$ at $30{ }^{\circ} \mathrm{C} .{ }^{18}{ }^{b}$ Recorded at $800 \mathrm{MHz}\left({ }^{1} \mathrm{H} \mathrm{NMR}\right)$ and $200 \mathrm{MHz}\left({ }^{13} \mathrm{C} \mathrm{NMR}\right)$ at $25{ }^{\circ} \mathrm{C}$. ${ }^{c}$ Not observed. ${ }^{d, e}$ overlap. 
Table 2: Biological data for serrulatanes $(\mathbf{1}-\mathbf{3})$ and the analogues $(\mathbf{6}-\mathbf{1 8})$

\begin{tabular}{|c|c|c|c|c|c|c|}
\hline \multirow[t]{2}{*}{ Compound } & & & & \multirow{2}{*}{$\begin{array}{l}\text { HEK293 } \\
\text { activity }^{c}\end{array}$} & \multirow{2}{*}{$\begin{array}{l}\text { Approximate } \\
\text { HEK293 IC }_{50}{ }^{d}\end{array}$} & \multirow[t]{2}{*}{ SI } \\
\hline & $\mathrm{IC}_{50} \pm \mathrm{SD}(\mu \mathrm{M})$ & $\mathrm{IC}_{50} \pm \mathrm{SD}(\mu \mathrm{M})$ & Selectivity ratio & & & \\
\hline 1 & $\mathrm{NA}^{* *}$ & NT & & & & NT \\
\hline 2 & $\mathrm{NA}^{* *}$ & NT & & & & NT \\
\hline 3 & $6.89 \pm 0.49$ & $8.76 \pm 0.25$ & 1.3 & 59 & $80 \mu \mathrm{M}$ & $\sim 11.61$ \\
\hline 6 & $1.40 \pm 0.90$ & $3.63 \pm 0.07$ & 2.6 & NA & $80 \mu \mathrm{M}$ & $>57$ \\
\hline 7 & $3.12 \pm 0.93$ & $7.42 \pm 0.74$ & 2.4 & NA & $80 \mu \mathrm{M}$ & $>26$ \\
\hline 8 & $2.70 \pm 3.04$ & $5.94 \pm 0.33$ & 2.2 & NA & $80 \mu \mathrm{M}$ & $>30$ \\
\hline 9 & $2.44 \pm 0.64$ & $4.57 \pm 0.56$ & 1.9 & 68 & $80 \mu \mathrm{M}$ & $\sim 32$ \\
\hline 10 & $1.25 \pm 0.35$ & $3.76 \pm 0.08$ & 3.0 & NA & $80 \mu \mathrm{M}$ & $>64$ \\
\hline 11 & $2.12 \pm 0.56$ & $6.17 \pm 1.33$ & 2.9 & 82 & $40 \mu \mathrm{M}$ & $\sim 19$ \\
\hline 12 & $1.42 \pm 0.12$ & $5.03 \pm 1.91$ & 3.6 & 71 & $40 \mu \mathrm{M}$ & $\sim 28$ \\
\hline 13 & $1.36 \pm 0.24$ & $4.96 \pm 1.41$ & 3.6 & 86 & $40 \mu \mathrm{M}$ & $\sim 29.4$ \\
\hline 14 & $1.79 \pm 0.55$ & $6.13 \pm 0.22$ & 3.4 & 44 & $80 \mu \mathrm{M}$ & $\sim 44$ \\
\hline 15 & $5.65 \pm 0.83$ & $8.76 \pm 0.55$ & 1.6 & $14.55 \mu \mathrm{M}$ & $14.55 \mu \mathrm{M}$ & 2.6 \\
\hline 16 & $9.97 \pm 1.61$ & $18.00 \pm 0.00$ & 1.8 & 100 & $40 \mu \mathrm{M}$ & $\sim 4$ \\
\hline 17 & $8.44 \pm 1.13$ & $10.16 \pm 0.48$ & 1.2 & 100 & $40 \mu \mathrm{M}$ & $\sim 4.7$ \\
\hline 18 & $13.62 \pm 6.77$ & NA & NA & 70 & $40 \mu \mathrm{M}$ & $\sim 2.9$ \\
\hline Chloroquine & $0.0053 \pm 0.0007$ & $0.0823 \pm 0.0349$ & 15.46 & NA* & $40 \mu \mathrm{M}$ & $>7512$ \\
\hline Artesunate & $0.0009 \pm 0.0001$ & $0.0010 \pm 0.0000$ & 0.82 & $91 * * *$ & $10 \mu \mathrm{M}$ & $>11641$ \\
\hline Puromycin & $0.0532 \pm 0.0035$ & $0.0438 \pm 0.0033$ & 1.16 & $0.46 \mu \mathrm{M}$ & $0.46 \mu \mathrm{M}$ & 9 \\
\hline
\end{tabular}

$3 \mathrm{D} 7^{\mathrm{a}}=P$. falciparum (chloroquine sensitive strain, $\mathrm{Dd} 2^{\mathrm{b}}=P$. falciparum (multidrug resistant) strain, $\mathrm{SD}=$ standard deviation. Dd2/3D7 $=$ resistance ratio. HEK $293^{\mathrm{c}}=$ human embryonic kidney cell line, average $\%$ inhibition at $80 \mu \mathrm{M} . \mathrm{NA}=$ not active at $80 \mu \mathrm{M} . \mathrm{NA} *=$ not active at $40 \mu \mathrm{M}$. NA** $=$ not active at $10 \mu \mathrm{M}$. $* * *=20 \mu \mathrm{M}$. Approximate HEK $\mathrm{IC}_{50}$ used for selectivity ${ }^{\mathrm{d}}=\mathrm{IC}_{50}$ value approximates allocated for each compound where an actual $\mathrm{IC}_{50}$ could not be determined due to inactivity at the tested concentration. SI = parasite selectivity ratio (approximated HEK293 $\mathrm{IC}_{50} /$ parasite actual $\mathrm{IC}_{50}$ ).

While the overall structure activity relationship (SAR) data for the amide analogues is somewhat limited, improved activity was observed when the carboxylic acid was converted to either phenethyl (16-10), benzyl (11-14) or aliphatic (15) amides with $\mathrm{IC}_{50}$ values ranging from $1.25-5.65 \mu \mathrm{M}$ and $3.63-8.76 \mu \mathrm{M}$ against 3D7 and Dd2, respectively. The 4-fluorophenethylamide analogue (10) was the most potent amide in the series with $\mathrm{IC}_{50}$ values of 1.25 and $3.76 \mu \mathrm{M}$ against $3 \mathrm{D} 7$ and $\mathrm{Dd} 2$ lines, respectively, demonstrating no common mechanism of resistance to this compound. This molecule also showed no activity at 80 $\mu \mathrm{M}$ towards HEK293, which translated to the best selectivity indices for this series $(>64)$. In order to obtain an indication of parasite selectivity over human HEK293 cell activity, where $\mathrm{IC}_{50}$ values for HEK293 activity were not obtainable, approximate $\mathrm{IC}_{50}$ values were assigned. Compounds with no activity at $80 \mu \mathrm{M}$ were assigned an $\mathrm{IC}_{50}$ value of $80 \mu \mathrm{M}$ and the SI expressed as greater than $(>)$. Compounds which demonstrated greater than $50 \%$ inhibitory activity at $80 \mu \mathrm{M}$ were assigned an $\mathrm{IC}_{50}$ value of $40 \mu \mathrm{M}$ and selectivity presented as approximately $(\sim)$. Compounds which demonstrated greater than $50 \%$ inhibitory activity at $80 \mathrm{uM}$ were assigned an $\mathrm{IC}_{50}$ value of $40 \mathrm{uM}$ and selectivity presented as approximately $(\sim)$. Interestingly, the only alkaloid synthesized during these studies, compound $\mathbf{1 5}$, display similar bioactivity to the other semi-synthetic analogues indicating that the antimalarial activity is not restricted to just the aryl series. Adding pivaloyl groups to the NP scaffold (1) improved the antimalarial activity as seen in compounds 16-18; potentially the increase of lipophilicity in the pivaloylated analogues may be responsible for this antiparasitic activity. The physicochemical properties of all the analogues generated were calculated (see supplementary material,
Table S1) using Cambridge Soft, ChemDraw ${ }^{\circledR}$ Ultra $^{31}$. While all compounds violated Lipinski's Rule of Five (Ro5) for physicochemical parameters $^{32}$ in relation to the MW (>500 Da) and $C \log P(>5)$ it was our intention to make the final screening library more Lipinski compliant by deprotecting the serrrulatane scaffold with a final base hydrolysis step. Removal of the pivaloyl groups from each of the amide analogues 6-15, would have resulted in significant reductions to CLogP and MW values (see supplementary material, Table S2) of the amide analogues and would have made all library members more drug-like. For example, compound 6 had calculated CLogP and MW values of 7.76 and $703 \mathrm{Da}$, respectively, however after pivaloyl removal the resulting product would have had values of 5.14 (CLogP) and 451 Da (MW), giving this molecule only one Lipinski Ro5 violation. Unfortunately, our attempts to generate the desired products proved unsuccessful using $\mathrm{NaOH}$ in acetone at $\mathrm{rt}$ for $16 \mathrm{~h}$, and due to the low yields of the pivaloylated amides $(\mathbf{6}-\mathbf{1 5})$ no further deprotection chemistry was attempted.

\section{Conclusion}

In summary, the plant-derived natural product scaffold, 3,7,8trihydroxyserrulat-14-en-19-oic acid (1) was utilized in the generation of an amide library via a one-pot mixed anhydride amidation using pivaloyl chloride. Preliminary antimalarial screening of $\mathbf{1}$ and the semi-synthetic analogues against 3D7 and Dd2 P. falciparum strains exhibited improved activity with compound $\mathbf{1 0}$ being most active and selective. These data identified scaffold $\mathbf{1}$ as a valuable starting point for the generation of unique amide analogues. This report further validates the use of isolated NPs for the generation of screening libraries. In this 
specific case a NP scaffold with no antimalarial activity was used to generate a series of new analogues, several of which displayed moderate anti-plasmodial activity and selectivity. Further antimalarial investigations of serrulatane NPs and analogues are warranted.

\section{Experimental section}

\subsection{General experimental procedures}

Optical rotations were determined on a JASCO P-1020 polarimeter. UV spectra were recorded on a JASCO V-650 $\mathrm{UV} / \mathrm{vis}$ spectrophotometer. NMR spectra were recorded in DMSO- $d_{6}$ at $25{ }^{\circ} \mathrm{C}$ on a Bruker Avance HDX $800 \mathrm{MHz}$ spectrometer equipped with a TCI cryoprobe. The ${ }^{1} \mathrm{H}$ and ${ }^{13} \mathrm{C}$ chemical shifts were referenced to the solvent peaks for DMSO- $d_{6}$ at $\delta_{\mathrm{H}} 2.50$ and $\delta_{\mathrm{C}} 39.5$, respectively. LRESIMS were recorded on a Waters ZQ mass spectrometer using a Phenomenex Luna $\mathrm{C}_{18}$ (2) $3 \mu \mathrm{m} 100 \AA(50 \times 4.60 \mathrm{~mm})$ column. HRESIMS data was acquired on a $12 \mathrm{~T}$ SolariX XR FT-ICR-MS. A Fritsch Pulverisette 19 Universal Cutting Mill was used to grind the plant material. An Edwards Instrument company Bio-line orbital shaker was used for plant extractions. A Waters 600 pump equipped with a Waters 966 PDA detector and a Gilson 715 liquid handler was used for semi-preparative separations. Alltech $\mathrm{C}_{18}$ bonded silica (35-75 $\mu \mathrm{m}, 150 \AA)$ and an Alltech stainless steel guard cartridge $(10 \times 30 \mathrm{~mm}$ or $35 \times 50 \mathrm{~mm})$ were used for pre-adsorption work. ThermoElectron $\mathrm{C}_{18}$ Betasil $5 \mu \mathrm{m} 143 \AA(150 \times 50 \mathrm{~mm}$ or $21.2 \times$ $150 \mathrm{~mm}$ ) columns were used for preparative and semi-preparative HPLC purifications of the NP scaffold (1). A Phenomenex Luna $\mathrm{C}_{18}(2) 5 \mu \mathrm{m} 100 \AA(250 \times 150 \mathrm{~mm})$ column was used to purify the reaction products. All solvents used for chromatography and MS were Lab Scan HPLC grade, and the $\mathrm{H}_{2} \mathrm{O}$ was Millipore Milli-Q PF filtered. All synthetic reagents were obtained from Sigma-Aldrich and used without further purification.

4.2 Plant material: E. microtheca was collected from South Brisbane, Queensland, Australia between April 2013 and December 2014. A voucher specimen (RAD039) has been deposited at the Eskitis Institute, Griffith University, Brisbane, Australia. The plant was air-dried, ground to a fine powder, and stored at rt prior to extraction.

4.3 Extraction and isolation: The air-dried and ground aerial parts of E. microtheca $(\sim 194 \mathrm{~g})$ were extracted with $\mathrm{CH}_{2} \mathrm{Cl}_{2}(3 \times$ $1 \mathrm{~L}$ ) at $\mathrm{rt}$ under constant shaking for $2 \mathrm{~h}$. The $\mathrm{CH}_{2} \mathrm{Cl}_{2}$ extract was dried under reduced pressure to yield a green gum $(25 \mathrm{~g})$. A portion $(5 \mathrm{~g})$ of the $\mathrm{CH}_{2} \mathrm{Cl}_{2}$ extract was pre-adsorbed to $\mathrm{C}_{18^{-}}$ bonded silica then packed into a guard cartridge $(35 \times 50 \mathrm{~mm})$ that was subsequently attached to a $\mathrm{C}_{18}$ Betasil $5 \mu \mathrm{m} 143 \AA$ (150 $\times 50 \mathrm{~mm}$ ) column and was subjected to preparative HPLC. Isocratic conditions of $50 \% \mathrm{H}_{2} \mathrm{O}(0.1 \% \mathrm{TFA}) / 50 \% \mathrm{MeOH}(0.1 \%$ TFA) were held for $10 \mathrm{~min}$, followed by a linear gradient to $\mathrm{MeOH}(0.1 \%$ TFA $)$ over $70 \mathrm{~min}$, then isocratic conditions of $\mathrm{MeOH}(0.1 \%$ TFA) for $10 \mathrm{~min}$, all at a flow rate of $20 \mathrm{~mL} / \mathrm{min}$. Ninety fractions $(90 \times 1 \mathrm{~min})$ were collected from the start of the HPLC run. The UV active fractions were analyzed by (-)LRESIMS and ${ }^{1} \mathrm{H}$ NMR; fractions containing compound 1 were pooled, dried and pre-adsorbed to $\mathrm{C}_{18}$-bonded silica then packed into a guard cartridge $(10 \times 30 \mathrm{~mm})$. This was attached to a $\mathrm{C}_{18}$ Betasil $5 \mu \mathrm{m} 143 \AA(21.2 \times 150 \mathrm{~mm})$ column and was subjected to semi-preparative HPLC. Isocratic conditions of $70 \% \mathrm{H}_{2} \mathrm{O}$ $(0.1 \%$ TFA $) / 30 \% \mathrm{MeOH}(0.1 \%$ TFA $)$ were held for $10 \mathrm{~min}$, followed by a linear gradient to $\mathrm{MeOH}(0.1 \%$ TFA) over $40 \mathrm{~min}$, then isocratic conditions of $\mathrm{MeOH}(0.1 \% \mathrm{TFA})$ for $10 \mathrm{~min}$, all at a flow rate of $9 \mathrm{~mL} / \mathrm{min}$. Sixty fractions $(60 \times 1 \mathrm{~min})$ were collected from the start of the HPLC run. Following multiple HPLC purifications, 3,7,8-trihydroxyserrulat-14-en-19-oic acid
(1, $800 \mathrm{mg}, 2.1 \%$ dry wt) was obtained. Compounds $\mathbf{2}$ and $\mathbf{3}$ were obtained from the Davis compound library for antimalarial assay. ${ }^{18}$

3,7,8-Trihydroxyserrulat-14-en-19-oic acid (1): light-brown gum, $(800 \mathrm{mg}, 2.1 \%)$. NMR, $[\boldsymbol{\alpha}]_{\mathrm{D}}$ and MS data were consistent with literature values. ${ }^{18}$

4.4 Generation of the amide library: Compound $\mathbf{1}(50 \mathrm{mg}$, $0.144 \mathrm{mmol})$ was dissolved in anhydrous pyridine $(500 \mu \mathrm{L})$ and pivaloyl chloride $(200 \mu \mathrm{L}, 0.0016 \mathrm{mmol})$ was added dropwise at $0{ }^{\circ} \mathrm{C}$ with constant stirring for $2 \mathrm{~h}$. The resulting mixture was allowed to warm to $\mathrm{rt}$ after which the desired amine $(1.44 \mathrm{mmol})$ was added and the mixture was stirred for a further $16 \mathrm{~h}$. The reaction crude was dried under $\mathrm{N}_{2}$ and then high vacuum before being pre-adsorbed to $\mathrm{C}_{18}(\sim 1 \mathrm{~g})$. This was then packed into a guard cartridge $(10 \times 30 \mathrm{~mm})$ that was subsequently attached to a Phenomenex Luna column for HPLC purification. Isocratic HPLC conditions of $50 \% \mathrm{H}_{2} \mathrm{O}(0.1 \%$ TFA $) / 50 \% \mathrm{MeOH}(0.1 \%$ TFA) were employed for the first $10 \mathrm{~min}$ followed by a linear gradient to $\mathrm{MeOH}(0.1 \%$ TFA) over $40 \mathrm{~min}$, and finally isocratic conditions of $\mathrm{MeOH}(0.1 \% \mathrm{TFA})$ for $10 \mathrm{~min}$, all at flow rate of 4 $\mathrm{mL} / \mathrm{min}$. Sixty fractions $(60 \times 1 \mathrm{~min})$ were collected from the start of the HPLC run. The UV active fractions were analyzed by LRESIMS followed by ${ }^{1} \mathrm{H}$ NMR spectroscopy to identify the desired amides.

Compound 6: white amorphous powder, (15.8 mg, 18\%); $[\alpha]_{\mathrm{D}}^{24}+24(c$ 0.1, MeOH); UV (MeOH) (log $\varepsilon) 216$ (4.17), 253 (3.68) nm; IR (UATR) $v_{\max } 2970,1757,1723,1656,1154,1094$, $1027 \mathrm{~cm}^{-1}$; ${ }^{1} \mathrm{H}$ NMR $\left(800 \mathrm{MHz}, \mathrm{DMSO}-d_{6}\right) \delta_{\mathrm{H}} 0.53(3 \mathrm{H}, \mathrm{d}, J=$ $6.8 \mathrm{~Hz}, \mathrm{H}-18), 1.14$ (9H, s, OPv-3), 1.20 (3H, overlap, H-20), $1.24(9 \mathrm{H}, \mathrm{s}, \mathrm{OPv}-7), 1.28(1 \mathrm{H}$, overlap, $\mathrm{H}-12 \mathrm{~b}), 1.31(9 \mathrm{H}, \mathrm{s}, \mathrm{OPv}-$ 8), $1.40(1 \mathrm{H}, \mathrm{m}, \mathrm{H}-12 \mathrm{a}), 1.57$ (3H, s, H-17), $1.61(1 \mathrm{H}, \mathrm{m}, \mathrm{H}-2 \beta)$, $1.65(3 \mathrm{H}, \mathrm{s}, \mathrm{H}-16), 1.95$ (1H, ddd, $J=7.9,7.8,7.8 \mathrm{~Hz}, \mathrm{H}-13 \mathrm{~b})$, 2.02 (1H, m, H-13a), 2.08 (1H, overlap, H-11), 2.10 (1H, overlap, H- $2 \alpha), 2.78(2 \mathrm{H}, \mathrm{m}, \mathrm{H}-23), 2.95(1 \mathrm{H}$, br m, H-1), 3.04 (1H, br d, $J$ $=4.5 \mathrm{~Hz}, \mathrm{H}-4), 3.34(1 \mathrm{H}, \mathrm{m}, \mathrm{H}-22 \mathrm{~b}), 3.39(1 \mathrm{H}, \mathrm{m}, \mathrm{H}-22 \mathrm{a}), 5.12$ $(1 \mathrm{H}, \mathrm{t}, J=7.0 \mathrm{~Hz}, \mathrm{H}-14), 5.20(1 \mathrm{H}, \mathrm{dt}, J=11.4,4.0 \mathrm{~Hz}, \mathrm{H}-3)$, $7.02(1 \mathrm{H}, \mathrm{s}, \mathrm{H}-5), 7.19(1 \mathrm{H}, \mathrm{dd}, J=7.4,7.4 \mathrm{~Hz}, \mathrm{H}-27), 7.23(2 \mathrm{H}$, d, $J=7.4 \mathrm{~Hz}, \mathrm{H}-25, \mathrm{H}-29), 7.28$ (2H, dd, $J=7.4,7.4 \mathrm{~Hz}, \mathrm{H}-26$, $\mathrm{H}-28), 8.43(1 \mathrm{H}, \mathrm{t}, J=5.6 \mathrm{~Hz}, \mathrm{NH}-21) ;{ }^{13} \mathrm{C}$ NMR $(200 \mathrm{MHz}$, DMSO- $\left.d_{6}\right) \delta_{\mathrm{C}} 17.5$ (C-17), 18.4 (C-18), 22.8 (C-20), 25.5 (C-16), 25.8 (C-13), 26.76 (OPv-3), $26.84(\mathrm{OPv}-7), 26.9$ (OPv-8), 28.2 (C-1), 31.0 (C-2), 31.0 (C-11), 34.9 (C-23), 37.7 (C-12), 38.3 (OPv-3), 38.5 (OPv-7), 38.6 (OPv-8), 40.7 (C-22), 45.7 (C-4), 68.8 (C-3), 124.2 (C-14), 126.00 (C-5), 126.04 (C-27), 128.5 (C6), 128.3 (C-26, C-28), 128.7 (C-25, C-29), 130.9 (C-15), 134.3 (C-10), 136.6 (C-9), 138.9 (C-7), 139.5 (C-24), 140.8 (C-8), 165.0 (C-19), 174.8 (OPv-7, OPv-8), 176.8 (OPv-3); (+)LRESIMS $m / z 704$ (100); (+)-HRESIMS $m / z 726.4335\left[\mathrm{M}+\mathrm{Na}^{+}\right.$ (calcd for $\mathrm{C}_{43} \mathrm{H}_{61} \mathrm{NO}_{7} \mathrm{Na}, 726.4340$ ).

Compound 7: white amorphous powder, $(2.7 \mathrm{mg}, 2 \%)$; $[\alpha]_{\mathrm{D}}^{24}$ +41 ( $c$ 0.1, MeOH); UV (MeOH) (log $\varepsilon) 224$ (4.22) nm; ${ }^{1} \mathrm{H}$ NMR $\left(800 \mathrm{MHz}, \mathrm{DMSO}-d_{6}\right) \delta_{\mathrm{H}} 0.53(3 \mathrm{H}, \mathrm{d}, J=6.8 \mathrm{~Hz}, \mathrm{H}-18), 1.14$ $(9 \mathrm{H}, \mathrm{s}, \mathrm{OPv}-3), 1.20(3 \mathrm{H}$, br overlap, H-20), $1.22(9 \mathrm{H}, \mathrm{s}, \mathrm{OPv}-8)$, $1.31(9 \mathrm{H}, \mathrm{s}, \mathrm{OPv}-7), 1.27(1 \mathrm{H}$, overlap, H-12b), $1.41(1 \mathrm{H}$, overlap, H-12a), 1.57 (3H, s, H-17), 1.61 (1H, overlap, H-2 $\beta$ ), $2.11(1 \mathrm{H}$, overlap, $\mathrm{H}-2 \alpha), 1.65(3 \mathrm{H}, \mathrm{s}, \mathrm{H}-16), 1.95(2 \mathrm{H}, \mathrm{m}, \mathrm{H}-$ 13b), 2.05 (2H, m, H-13a), 2.06 (1H, overlap, H-11), 2.75 (2H, m, H-23), 2.95 (1H, br m, H-1), 3.03 (1H, br s, H-4), 3.32 (1H, m, H22b), 3.39 (1H, m, H-22a), 5.13 (1H, t, $J=6.4 \mathrm{~Hz}, \mathrm{H}-14), 5.19$ $(1 \mathrm{H}, \mathrm{dt}, J=11.4,4.0 \mathrm{~Hz}, \mathrm{H}-3), 6.99(1 \mathrm{H}, \mathrm{s}, \mathrm{H}-5), 7.20(2 \mathrm{H}, \mathrm{m}, \mathrm{H}-$ 29), 7.45 (2H, m, H-26, H-28), 8.40 (1H, t, $J=5.6 \mathrm{~Hz}, \mathrm{NH}-21)$; ${ }^{13} \mathrm{C}$ NMR $\left(200 \mathrm{MHz}, \mathrm{DMSO}-d_{6}\right) \delta_{\mathrm{C}} 17.5$ (C-17), 18.4 (C-18), 22.7 (C-20), 25.5 (C-16), 25.8 (C-13), 26.75 (OPv-3), 26.83 (OPv-7), 26.9 (OPv-8), 28.1 (C-1), 31.0 (C-2), 31.0 (C-11), 34.1 (C-23), 
37.7 (C-12), 38.3 (OPv-3), 38.4 (OPv-8), $38.6(\mathrm{OPv}-7), 40.3$ (C22), 45.7 (C-4), 68.8 (C-3), 119.2 (C-27), 124.1 (C-14), 126.0 (C5), 128.3 (C-6), 130.9 (C-15), 131.1 (C-25, C-29), 131.0 (C-26, C-28), 134.4 (C-10), 136.6 (C-9), 138.9 (C-7), 139.0 (C-24), 140.8 (C-8), 165.0 (C-19), 174.8 (OPv-7, OPv-8), 176.8 (OPv-3); $(+)$-LRESIMS $\left.m / z 782(98)\left[{ }^{79} \mathrm{Br}: \mathrm{M}+\mathrm{H}\right]\right]^{+}, 784(100)\left[{ }^{81} \mathrm{Br}: \mathrm{M}+\right.$ $\mathrm{H}]^{+}$; (+)-HRESIMS $\mathrm{m} / \mathrm{z} 804.3441\left[\mathrm{M}+\mathrm{Na}^{+}\right.$(calcd for $\left.\mathrm{C}_{43} \mathrm{H}_{60}{ }^{79} \mathrm{BrNO}_{7} \mathrm{Na}, 804.3445\right)$.

Compound 8: white amorphous powder, $(7.9 \mathrm{mg}, 8 \%)$; $[\alpha]_{\mathrm{D}}^{24}$ +26 (c 0.1, MeOH); UV (MeOH) (log $\varepsilon) 208$ (4.99), 225 (4.67), $277(3.36) \mathrm{nm} ;{ }^{1} \mathrm{H}$ NMR $\left(800 \mathrm{MHz}, \mathrm{DMSO}-d_{6}\right) \delta_{\mathrm{H}} 0.53(3 \mathrm{H}, \mathrm{d}, J=$ $6.9 \mathrm{~Hz}, \mathrm{H}-18), 1.14$ (9H, s, OPv-3), 1.22 (9H, s, OPv-8), 1.19 (3H, overlap, H-20), 1.27 (1H, overlap, H-12b), $1.31(9 \mathrm{H}, \mathrm{s}, \mathrm{OPv}-$ 7), $1.40(1 \mathrm{H}$, overlap, H-12a), $1.57(3 \mathrm{H}, \mathrm{s}, \mathrm{H}-17), 1.61(1 \mathrm{H}, \mathrm{m}, \mathrm{H}-$ 2ß), $1.64(3 \mathrm{H}, \mathrm{s}, \mathrm{H}-16), 1.95(1 \mathrm{H}, \mathrm{m}, \mathrm{H}-13 \mathrm{~b}), 2.03(1 \mathrm{H}, \mathrm{m}, \mathrm{H}-$ 13a), 2.08 (1H, overlap, H-11), $2.10(1 \mathrm{H}, \mathrm{m}, \mathrm{H}-2 \alpha), 2.77(2 \mathrm{H}, \mathrm{m}$, H-23), 2.95 (1H, br m, H-1), $3.03(1 \mathrm{H}$, br d, $J=3.8 \mathrm{~Hz}, \mathrm{H}-4)$, $3.36(1 \mathrm{H}, \mathrm{m}, \mathrm{H}-22 \mathrm{~b}), 3.38(1 \mathrm{H}, \mathrm{m}, \mathrm{H}-22 \mathrm{a}), 5.12(1 \mathrm{H}, \mathrm{t}, J=7.0, \mathrm{H}-$ 14), $5.19(1 \mathrm{H}, \mathrm{dt}, J=11.5,4.0 \mathrm{~Hz}, \mathrm{H}-3), 7.00(1 \mathrm{H}, \mathrm{s}, \mathrm{H}-5), 7.26$ (2H, m, H-25, H-29), 7.32 (2H, m, H-26, H-28), 8.40 (1H, t $J=$ $5.7 \mathrm{~Hz}, \mathrm{NH}-21) ;{ }^{13} \mathrm{C}$ NMR $\left(200 \mathrm{MHz}, \mathrm{DMSO}-d_{6}\right) \delta_{\mathrm{C}} 18.0(\mathrm{C}-17)$, 18.9 (C-18), 23.2 (C-20), 26.0 (C-16), 26.3 (C-13), 27.30 (OPv8), $27.2(\mathrm{OPv}-3), 27.34(\mathrm{OPv}-7), 28.6(\mathrm{C}-1), 31.5(\mathrm{C}-2), 31.5,(\mathrm{C}-$ 11), 34.5 (C-23), 38.2 (C-12), 38.8 (OPv-3), 38.9 (OPv-8), 39.1 (OPv-7), 40.8 (C-22), 46.1 (C-4), 69.3 (C-3), 124.6 (C-14), 126.4 (C-5), 128.6 (C-26, C-28), 128.8 (C-6), 131.0 (C-25, C-29), 131.1 (C-15), 134.8 (C-10), 137.1 (C-9), 139.0 (C-24), 139.4 (C-7), 141.2 (C-8), 165.4 (C-19), $175.2(\mathrm{OPv}-7, \mathrm{OPv}-8), 177.3$ (OPv-3); (+)-LRESIMS $\left.m / z 738(100)\left[{ }^{35} \mathrm{Cl}: \mathrm{M}+\mathrm{H}\right]\right]^{+}, 740(33)\left[{ }^{37} \mathrm{Cl}: \mathrm{M}+\right.$ $\mathrm{H}]^{+}$; (-)-LRESIMS $m / z 736(100)\left[{ }^{35} \mathrm{Cl}: \mathrm{M}-\mathrm{H}\right]{ }^{-}, 738(33)\left[{ }^{37} \mathrm{Cl}\right.$ : $\mathrm{M}-\mathrm{H}]^{-} ;(+)$-HRESIMS $m / z 760.3939[\mathrm{M}+\mathrm{Na}]^{+}$(calcd for $\left.\mathrm{C}_{43} \mathrm{H}_{60}{ }^{35} \mathrm{ClNO}_{7} \mathrm{Na}, 760.3951\right)$.

Compound 9: white amorphous powder, (10.1 mg, 12\%); $[\alpha]_{\mathrm{D}}^{24}+12(c$ 0.1, MeOH); UV (MeOH) $(\log \varepsilon) 209$ (5.01), 277 (3.54) nm; ${ }^{1} \mathrm{H}$ NMR $\left(800 \mathrm{MHz}, \mathrm{DMSO}-d_{6}\right) \delta_{\mathrm{H}} 0.38(3 \mathrm{H}, \mathrm{d}, J=$ 7.0, H-18), $1.22(9 \mathrm{H}, \mathrm{s}, \mathrm{OPv}-8), 1.14(3 \mathrm{H}$, overlap, H-20), 1.31 $(9 \mathrm{H}, \mathrm{s}, \mathrm{OPv}-7), 1.32(1 \mathrm{H}$, overlap, H-12b), $1.52(1 \mathrm{H}$, overlap, $\mathrm{H}-$ 12a), 1.52 (2H, m, H-23), 1.59 (3H, s, H-17), 1.66 (3H, s, H-16), $1.88(2 \mathrm{H}, \mathrm{m}, \mathrm{H}-2 \alpha), 2.01(2 \mathrm{H}, \mathrm{m}, \mathrm{H}-13), 2.15(1 \mathrm{H}, \mathrm{m}, \mathrm{H}-11), 2.77$ $(2 \mathrm{H}, \mathrm{m}, \mathrm{H}-23), 2.85(1 \mathrm{H}$, br m, H-1), $2.81(1 \mathrm{H}$, br d, $J=4.0 \mathrm{~Hz}$, $\mathrm{H}-4), 3.33$ (1H, m, H-22b), $3.38(1 \mathrm{H}, \mathrm{m}, \mathrm{H}-22 \mathrm{a}), 5.17(1 \mathrm{H}, \mathrm{t}, J=$ 7.0, H-14), 4.10 (1H, dt, $J=11.0,4.0 \mathrm{~Hz}, \mathrm{H}-3), 6.93(1 \mathrm{H}, \mathrm{s}, \mathrm{H}-5)$, 7.25 (2H, m, H-25, H-29), 7.32 (2H, m, H-26, H-28), $8.37(1 \mathrm{H}, \mathrm{t}$, $J=5.6 \mathrm{~Hz}, \mathrm{NH}-21) ;{ }^{13} \mathrm{C}$ NMR $\left(200 \mathrm{MHz}, \mathrm{DMSO}-d_{6}\right) \delta_{\mathrm{C}} 17.6(\mathrm{C}-$ 17), 18.9 (C-18), 23.2 (C-20), 25.6 (C-16), 25.8 (C-13), 26.8 (OPv-8), 27.9 (OPv-7), 28.7 (C-1), 29.9 (C-11), 33.9 (C-2), 34.0 (C-23), 38.4 (OPv-8), 38.6 (C-12), $38.6(\mathrm{OPv}-7), 40.3$ (C-22), 48.6 (C-4), 64.3 (C-3), 124.8 (C-14), 126.4 (C-5), 128.0 (C-6), 128.1 (C, C-26, C-28), 130.4 (C-15), 130.6 (C-25, C-29), 130.7 (C-27), 136.1 (C-9), 136.9 (C-10), 138.5 (C-7), 138.6 (C-24), 140.8 (C-8), 165.1 (C-19), 174.8 (OPv-7, OPv-8); (+)-LRESIMS $m / z 654$ (100) $\left[{ }^{35} \mathrm{Cl}: \mathrm{M}+\mathrm{H}\right]^{+}, 656(33)\left[{ }^{37} \mathrm{Cl}: \mathrm{M}+\mathrm{H}\right]^{+} ;(-)$ LRESIMS $m / z 652(100)\left[{ }^{35} \mathrm{Cl}: \mathrm{M}-\mathrm{H}\right]^{-}, 654(33)\left[{ }^{37} \mathrm{Cl}: \mathrm{M}-\mathrm{H}\right]^{-}$; $(+)$-HRESIMS $m / z \quad 676.3364 \quad\left[\mathrm{M}+\mathrm{Na}^{+}\right.$(calcd for $\left.\mathrm{C}_{38} \mathrm{H}_{52}{ }^{35} \mathrm{ClNO}_{6} \mathrm{Na}, 676.3375\right)$.

Compound 10: white amorphous powder, $(6.7 \mathrm{mg}, 6 \%)$; $[\alpha]_{\mathrm{D}}^{24}$ +14 (c 0.1, MeOH); UV (MeOH) (log $\varepsilon) 210$ (5.10), 239 (4.37), $272(3.65) \mathrm{nm} ;{ }^{1} \mathrm{H}$ NMR $\left(800 \mathrm{MHz}, \mathrm{DMSO}-d_{6}\right) \delta_{\mathrm{H}} 0.52(3 \mathrm{H}, \mathrm{d}, J$ $=7.0 \mathrm{~Hz}, \mathrm{H}-18), 1.14(9 \mathrm{H}, \mathrm{s}, \mathrm{OPv}-3), 1.19(3 \mathrm{H}$, overlap, H-20), $1.23(9 \mathrm{H}, \mathrm{s}, \mathrm{OPv}-7), 1.27(1 \mathrm{H}, \mathrm{m}, \mathrm{H}-12 \mathrm{a}), 1.31(9 \mathrm{H}, \mathrm{s}, \mathrm{OPv}-8)$, $1.40(1 \mathrm{H}, \mathrm{m}, \mathrm{H}-12 \mathrm{~b}), 1.57(3 \mathrm{H}, \mathrm{s}, \mathrm{H}-17), 1.61(1 \mathrm{H}, \mathrm{m}, \mathrm{H}-2 \beta), 1.64$ (3H, s, H-16), $1.95(1 \mathrm{H}, \mathrm{m}, \mathrm{H}-13 \mathrm{~b}), 2.01(1 \mathrm{H}$, overlap, H-13a), $2.07(1 \mathrm{H}$, overlap, $\mathrm{H}-11), 2.10(1 \mathrm{H}, \mathrm{m}, \mathrm{H}-2 \alpha), 2.76(2 \mathrm{H}, \mathrm{m}, \mathrm{H}-$ 23), $2.96(1 \mathrm{H}$, br m, H-1), $3.04(1 \mathrm{H}$, br d, $4.5 \mathrm{~Hz}, \mathrm{H}-4), 3.32(1 \mathrm{H}$, m, H-22b), 3.38 (1H, m, H-22a), $5.11(1 \mathrm{H}, \mathrm{t}, J=7.0 \mathrm{~Hz}, \mathrm{H}-14)$, $5.19(1 \mathrm{H}, \mathrm{dt}, J=11.0,4.0 \mathrm{~Hz}, \mathrm{H}-3), 7.00(1 \mathrm{H}, \mathrm{s}, \mathrm{H}-5), 7.08(2 \mathrm{H}$, m, H-26, H-28), 7.26 (2H, m, H-25, H-29), 8.40 (1H, t, $J=6.0$ $\mathrm{Hz}, \mathrm{NH}-21) ;{ }^{13} \mathrm{C}$ NMR $\left(200 \mathrm{MHz}, \mathrm{DMSO}-d_{6}\right) \delta_{\mathrm{C}} 17.5(\mathrm{C}-17)$, 18.4 (C-18), 22.7 (C-20), 25.5 (C-16), 25.8 (C-13), 26.7 (OPv-3), 26.8 (OPv-7), 26.9 (OPv-8), 28.2 (C-1), 31.0 (C-2), 31.0 (C-11), 33.9 (C-23), 37.7 (C-12), 38.3 (OPv-8), 38.4 (OPv-3), 38.6 (OPv7), $40.6(\mathrm{C}-22), 45.6(\mathrm{C}-4), 68.8(\mathrm{C}-3), 114.9\left(\mathrm{~d},{ }^{2} J_{\mathrm{CF}}=20.6 \mathrm{~Hz}\right.$, C-26, C-28), 124.1 (C-14), 126.0 (C-5), 128.4 (C-6), 130.5 (d, $\left.{ }^{3} J_{\mathrm{CF}}=7.5 \mathrm{~Hz} \mathrm{C}-25, \mathrm{C}-29\right), 130.9$ (C-15), 134.3 (C-10), 135.6 (C24), 136.6 (C-9), 138.9 (C-7), 140.8 (C-8), 160.8 (d, ${ }^{1} J_{\mathrm{CF}}=242.0$ $\mathrm{Hz}, \mathrm{C}-27), 165.0$ (C-19), 174.8 (OPv-7, OPv-8), 176.8 (OPv-3); $(+)$-LRESIMS $m / z 722(100)[\mathrm{M}+\mathrm{H}]^{+} ;(+)$-HRESIMS $\mathrm{m} / \mathrm{z}$ $722.4425[\mathrm{M}+\mathrm{H}]^{+}$(calcd for $\mathrm{C}_{43} \mathrm{H}_{61} \mathrm{FNO}_{7}, 722.4427$ ).

Compound 11: white amorphous powder, $(5.3 \mathrm{mg}, 5 \%)$; $[\alpha]_{\mathrm{D}}^{24}$ +8 (c 0.1, MeOH); UV (MeOH) (log $\varepsilon) 208$ (4.69), 297 (2.88) $\mathrm{nm} ;{ }^{1} \mathrm{H}$ NMR $\left(800 \mathrm{MHz}, \mathrm{DMSO}-d_{6}\right) \delta_{\mathrm{H}} 0.57(3 \mathrm{H}, \mathrm{d}, J=7.0 \mathrm{~Hz}$, $\mathrm{H}-18), 1.13(9 \mathrm{H}, \mathrm{s}, \mathrm{OPv}-3), 1.18(9 \mathrm{H}, \mathrm{s}, \mathrm{OPv}-7), 1.20(3 \mathrm{H}$, overlap, H-20), 1.29 (1H, m, H-12b), $1.32(9 \mathrm{H}, \mathrm{s}, \mathrm{OPv}-8), 1.42$ $(1 \mathrm{H}, \mathrm{m}, \mathrm{H}-12 \mathrm{a}), 1.55$ (3H, s, H-17), 1.62 (1H, overlap, H-2 $\beta)$, $1.64(3 \mathrm{H}, \mathrm{s}, \mathrm{H}-16), 1.95(1 \mathrm{H}, \mathrm{m}, \mathrm{H}-13 \mathrm{~b}), 2.01(1 \mathrm{H}$, overlap, H13a), 2.08 (1H, overlap, H-11), 2.14 (1H, overlap, H-2 $\alpha), 2.98$ $(1 \mathrm{H}$, br m, H-1), 3.08 (1H, br s, H-4), $4.34(1 \mathrm{H}, \mathrm{dd}, J=15.1,6.1$ $\mathrm{Hz}, \mathrm{H}-22 \mathrm{~b}), 4.41(1 \mathrm{H}, \mathrm{dd}, J=15.1,6.1 \mathrm{~Hz}, \mathrm{H}-22 \mathrm{a}), 5.10(1 \mathrm{H}, \mathrm{t}, J$ $=7.0 \mathrm{~Hz}, \mathrm{H}-14), 5.21(1 \mathrm{H}, \mathrm{dt}, J=11.0,4.0 \mathrm{~Hz}, \mathrm{H}-3), 7.18(1 \mathrm{H}, \mathrm{s}$, H-5), 7.23 (1H, m, H-26), 7.29 (2H, m, H-25, H-27), 7.30 (2H, m, $\mathrm{H}-24, \mathrm{H}-28), 8.91(1 \mathrm{H}, \mathrm{t}, J=6.1 \mathrm{~Hz}, \mathrm{NH}-21) ;{ }^{13} \mathrm{C}$ NMR $(200$ MHz, DMSO- $\left.d_{6}\right) \delta_{\mathrm{C}} 17.5$ (C-17), 18.4 (C-18), 22.8 (C-20), 25.5 (C-16), 25.8 (C-13), 26.9 (OPv-3), 26.8 (OPv-7), 26.8 (OPv-8), 28.4 (C-1), 31.1 (C-11), 31.2 (C-2), 37.4 (C-12), 38.3 (OPv-7), 38.4 (OPv-3), 38.6 (OPv-8), 42.3 (C-22), 45.5 (C-4), 68.8 (C-3), 124.1 (C-14), 126.0 (C-5), 126.7 (C-26), 127.2 (C-24, C-28), 128.1 (C-25, C-27), 128.2 (C-6), 130.9 (C-15), 134.4 (C-10), 136.8 (C-9), 139.1 (C-7), 139.3 (C-23), 140.9 (C-8), 165.0 (C19), $174.8(\mathrm{OPv}-7, \mathrm{OPv}-8), 176.8(\mathrm{OPv}-3) ;(+)$-LRESIMS $\mathrm{m} / \mathrm{z}-$ $690(100)[\mathrm{M}+\mathrm{H}]^{+} ;(+)$-HRESIMS $m / z 712.4185[\mathrm{M}+\mathrm{Na}]^{+}$ (calcd for $\mathrm{C}_{42} \mathrm{H}_{59} \mathrm{NO}_{7} \mathrm{Na}, 712.4184$ ).

Compound 12: white amorphous powder, $(1.8 \mathrm{mg}, 2 \%)$; [ $[\boldsymbol{\alpha}]_{\mathrm{D}}^{24}$ +22 (c 0.1, MeOH); UV (MeOH) (log $\varepsilon) 208$ (0.36), 229 (3.74) $\mathrm{nm} ;{ }^{1} \mathrm{H}$ NMR $\left(800 \mathrm{MHz}, \mathrm{DMSO}-d_{6}\right) \delta_{\mathrm{H}} 0.56(3 \mathrm{H}, \mathrm{d}, J=6.8 \mathrm{~Hz}$, $\mathrm{H}-18), 1.13(9 \mathrm{H}, \mathrm{s}, \mathrm{OPv}-3), 1.16(9 \mathrm{H}, \mathrm{s}, \mathrm{OPv}-8), 1.20(3 \mathrm{H}$, overlap, H-20), 1.29 (1H, overlap, H-12b), 1.31 (9H, OPv-7), $1.41(1 \mathrm{H}$, overlap, H-12a), $1.55(3 \mathrm{H}, \mathrm{s}, \mathrm{H}-17), 1.62(1 \mathrm{H}$, overlap, H-2ß), 1.64 (3H, s, H-16), 1.95 (1H, m, H-13b), 2.01 (1H, m, H13a), $2.08(1 \mathrm{H}$, overlap, H-11), $2.13(1 \mathrm{H}$, overlap, $\mathrm{H}-2 \alpha), 2.95$ $(1 \mathrm{H}$, br m, H-1), $3.08(1 \mathrm{H}, \mathrm{m}, \mathrm{H}-4), 4.29(1 \mathrm{H}, \mathrm{dd}, J=15.3,6.0$ $\mathrm{Hz}, \mathrm{H}-22 \mathrm{~b}), 4.37$ (1H, $J=\mathrm{dd}, 15.3,6.0 \mathrm{~Hz}, \mathrm{H}-22 \mathrm{a}), 5.10(1 \mathrm{H}, \mathrm{t}$, $7.0 \mathrm{~Hz}, \mathrm{H}-14), 5.21(1 \mathrm{H}, \mathrm{dt}, 11.0,4.0 \mathrm{~Hz}, \mathrm{H}-3), 7.16(1 \mathrm{H}, \mathrm{s}, \mathrm{H}-5)$, 7.26 (2H, m, H-24, H-28), 7.48 (2H, m, H-25, H-27), 8.93 (1H, t, $J=6.0 \mathrm{~Hz}, \mathrm{NH}-21) ;{ }^{13} \mathrm{C}$ NMR $\left(200 \mathrm{MHz}, \mathrm{DMSO}-d_{6}\right) \delta_{\mathrm{C}} 17.5(\mathrm{C}-$ 17), 18.4 (C-18), 22.8 (C-20), 25.5 (C-16), 25.8 (C-13), 26.75 (OPv-3), 26.81 (OPv-8), 26.84 (OPv-7), 28.1 (C-1), 31.1 (C-11), 31.2 (C-2), 37.4 (C-12), $38.3(\mathrm{OPv}-3), 38.4(\mathrm{OPv}-8), 38.6(\mathrm{OPv}-$ 7), 41.8 (C-22), 45.5 (C-4), 68.8 (C-3), 119.7 (C-26), 124.1 (C14), 126.0 (C-5), 128.0 (C-6), 129.5 (C-24, C-28), 131.0 (C-15), ${ }^{*}$ 131.0 (C-25, C-27), ${ }^{*} 134.4$ (C-10), 137.0 (C-9), 138.8 (C-23), 138.8 (C-7), ${ }^{*} 139.0$ (C-8), 165.1 (C-19), 174.8 (OPv-7, OPv-8), 176.8 (OPv-3); (+)-LRESIMS m/z 768 (98) $\left[{ }^{79} \mathrm{Br}: \mathrm{M}+\mathrm{H}\right]^{+}, 770$ (100) $\left[{ }^{81} \mathrm{Br}: \mathrm{M}+\mathrm{H}\right]^{+}$; (-)-LRESIMS $m / z 766$ (98) $\left[{ }^{79} \mathrm{Br}: \mathrm{M}-\mathrm{H}\right]^{-}$, 768 (98), [ $\left.{ }^{81} \mathrm{Br}: \mathrm{M}-\mathrm{H}\right]^{-}$; (+)-HRESIMS $m / z 790.3291[\mathrm{M}+\mathrm{Na}]^{+}$ (calcd for $\mathrm{C}_{42} \mathrm{H}_{58}{ }^{79} \mathrm{BrNO}_{7}, 790.3289$ ). "Interchangeable signals.

Compound 13: white amorphous powder, $(4.3 \mathrm{mg}, 4 \%)$; $[\alpha]_{\mathrm{D}}^{24}$ +15 (c 0.1, MeOH); UV (MeOH) (log $\varepsilon) 207$ (0.11), 224 (4.13) $\mathrm{nm} ;{ }^{1} \mathrm{H}$ NMR $\left(800 \mathrm{MHz}, \mathrm{DMSO}-d_{6}\right) \delta_{\mathrm{H}} 0.56(3 \mathrm{H}, \mathrm{d}, J=\mathrm{H}-18)$, 
$1.13(9 \mathrm{H}, \mathrm{s}, \mathrm{OPv}-3), 1.16(9 \mathrm{H}, \mathrm{s}, \mathrm{OPv}-7), 1.21$ (3H, overlap, H20), $1.28(1 \mathrm{H}, \mathrm{m}, \mathrm{H}-12 \mathrm{~b}), 1.31(9 \mathrm{H}, \mathrm{s}, \mathrm{OPv}-8), 1.40(1 \mathrm{H}, \mathrm{m}, \mathrm{H}-$ 12a), $1.55(3 \mathrm{H}, \mathrm{s}, \mathrm{H}-17), 1.61(1 \mathrm{H}$, overlap, $\mathrm{H}-2 \beta), 1.64(3 \mathrm{H}, \mathrm{s}$, H-16), 1.94 (1H, overlap, H-13b), 2.01 (1H, overlap, H-13a), 2.08 (1H, overlap, H-11), 2.10 (1H, overlap, H-2 $\alpha$ ), 2.93 (1H, br m, H1), $3.08(1 \mathrm{H}, \mathrm{m}, \mathrm{H}-4), 4.31(1 \mathrm{H}, \mathrm{dd}, J=15.3,6.0, \mathrm{H}-22 \mathrm{~b}), 4.39$ $(1 \mathrm{H}, \mathrm{dd}, J=15.3,6.0, \mathrm{H}-22 \mathrm{a}), 5.10(1 \mathrm{H}, \mathrm{t}, J=7, \mathrm{H}-14), 5.21(1 \mathrm{H}$, $\mathrm{dt}, J=11.0,4.0 \mathrm{~Hz}, \mathrm{H}-3), 7.16(1 \mathrm{H}, \mathrm{s}, \mathrm{H}-5), 7.32(2 \mathrm{H}, \mathrm{m}, \mathrm{H}-24$ $\mathrm{H}-28), 7.35$ (2H, m, H-25, H-27), $8.93(1 \mathrm{H}, \mathrm{t}, J=6.1 \mathrm{~Hz}, \mathrm{NH}-$ $21) ;{ }^{13} \mathrm{C}$ NMR $\left(200 \mathrm{MHz}, \mathrm{DMSO}-d_{6}\right) \delta_{\mathrm{C}} 17.5(\mathrm{C}-17), 18.4(\mathrm{C}-18)$, 22.8 (C-20), 25.5 (C-16), 25.8 (C-13), 26.8 (OPv-3), 26.8 (OPv$7),{ }^{*} 26.8(\mathrm{OPv}-8),{ }^{*} 28.2(\mathrm{C}-1), 31.2(\mathrm{C}-2, \mathrm{C}-11), 37.4(\mathrm{C}-12), 38.3$ $(\mathrm{OPv}-3), 38.4(\mathrm{OPv}-7), 38.7(\mathrm{OPv}-8), 41.8(\mathrm{C}-22), 45.5(\mathrm{C}-4)$, 68.8 (C-3), 124.2 (C-14), 126.0 (C-5), 128.1 (C-25, C-27), 129.1 (C-24, C-28), 131.0 (C-6, C-15), 131.3 (C-26), 134.5 (C-10), 138.4 (C-23), 137.0 (C-9), 139.1 (C-7), 140.9 (C-8), 165.1 (C19), $174.9(\mathrm{OPv}-7, \mathrm{OPv}-8), 176.9(\mathrm{OPv}-3)$; (+)-LRESIMS $\mathrm{m} / \mathrm{z}$ $724(100)\left[{ }^{35} \mathrm{Cl}: \mathrm{M}+\mathrm{H}\right]^{+}, 726(33)\left[{ }^{37} \mathrm{Cl}: \mathrm{M}+\mathrm{H}\right]^{+} ;(-)$-LRESIMS m/z 722 (100), $\left[{ }^{35} \mathrm{Cl}: \mathrm{M}-\mathrm{H}\right]^{-}, 724$ (33) $\left[{ }^{37} \mathrm{Cl}: \mathrm{M}-\mathrm{H}\right]^{-}$; (+)HRESIMS $m / z 746.3795[\mathrm{M}+\mathrm{Na}]^{+}$(calcd for $\mathrm{C}_{42} \mathrm{H}_{58}{ }^{35} \mathrm{ClNO}_{7} \mathrm{Na}$, 746.3794). " Interchangeable signals.

Compound 14: white amorphous powder, $(4 \mathrm{mg}, 4 \%)$; $[\alpha]_{\mathrm{D}}^{24}$ +18 (c 0.1, MeOH); UV (MeOH) (log $\varepsilon) 214$ (4.01), 241 (3.52), $306(3.19) \mathrm{nm} ;{ }^{1} \mathrm{H}$ NMR $\left(800 \mathrm{MHz}, \mathrm{DMSO}-d_{6}\right) \delta_{\mathrm{H}} 0.56(3 \mathrm{H}, \mathrm{d}, J$ $=7.0 \mathrm{~Hz}, \mathrm{H}-18), 1.13(9 \mathrm{H}, \mathrm{s}, \mathrm{OPv}-3), 1.16(9 \mathrm{H}, \mathrm{s}, \mathrm{OPv}-7), 1.20$ (3H, overlap, H-20), 1.28 (1H, m, H-12b), 1.31 (9H, s, OPv-8), $1.41(1 \mathrm{H}, \mathrm{m}, \mathrm{H}-12 \mathrm{a}), 1.55(3 \mathrm{H}, \mathrm{s}, \mathrm{H}-17), 1.62(1 \mathrm{H}$, overlap, $\mathrm{H}-$ 2B), $1.63(3 \mathrm{H}, \mathrm{s}, \mathrm{H}-16), 1.95(1 \mathrm{H}, \mathrm{m}, \mathrm{H}-13 \mathrm{~b}), 2.00(1 \mathrm{H}, \mathrm{m}, \mathrm{H}-$ 13a), $2.08(1 \mathrm{H}, \mathrm{m}, \mathrm{H}-11), 2.12(1 \mathrm{H}$, overlap, $\mathrm{H}-2 \alpha), 2.98(1 \mathrm{H}, \mathrm{br}$ s, H-1), $3.08(1 \mathrm{H}, \mathrm{m} \mathrm{H}-4), 4.30(1 \mathrm{H}, \mathrm{dd}, J=15.0,6.0 \mathrm{~Hz}, \mathrm{H}-22 \mathrm{~b})$, $4.39(1 \mathrm{H}, \mathrm{dd}, J=15.0,6.0 \mathrm{~Hz}, \mathrm{H}-22 \mathrm{a}), 5.10(1 \mathrm{H}, \mathrm{t}, J=7.0 \mathrm{~Hz}, \mathrm{H}-$ 14), $5.21(1 \mathrm{H}, \mathrm{dt}, J=11.0,4.0 \mathrm{~Hz}, \mathrm{H}-3), 7.11(2 \mathrm{H}, \mathrm{m}, \mathrm{H}-25, \mathrm{H}-$ 27), 7.16 (1H, s, H-5), 7.34 (2H, m, H-24, H-28), 8.91 (1H, t, $J=$ $6.0 \mathrm{~Hz}, \mathrm{NH}-21) ;{ }^{13} \mathrm{C}$ NMR $\left(200 \mathrm{MHz}, \mathrm{DMSO}-d_{6}\right) \delta_{\mathrm{C}} 17.5(\mathrm{C}-17)$, 18.4 (C-18), 22.8 (C-20), 25.5 (C-16), 25.8 (C-13), 26.84 (OPv$3),{ }^{*} 26.81(\mathrm{OPv}-7),{ }^{*} 26.7(\mathrm{OPv}-8),{ }^{*} 28.2(\mathrm{C}-1), 31.1$ (C-11), 31.2 (C-2), 37.4 (C-12), 38.4 (OPv-3), * 38.4 (OPv-7), 38.7 (OPv-8), 41.7 (C-22), 45.5 (C-4), $68.8(\mathrm{C}-3), 114.7\left(\mathrm{~d},{ }^{2} J_{\mathrm{CF}}=21.1 \mathrm{~Hz}, \mathrm{C}-\right.$ 25, C-27), 124.1 (C-14), 126.0 (C-5), $128.1(\mathrm{C}-6), 129.2\left(\mathrm{~d},{ }^{3} J_{\mathrm{CF}}=\right.$ $7.5 \mathrm{~Hz}, \mathrm{C}-24, \mathrm{C}-28), 130.9$ (C-15), 134.4 (C-10), $135.5\left(\mathrm{~d},{ }^{4} J_{\mathrm{CF}}=\right.$ $2.8 \mathrm{~Hz}, \mathrm{C}-23$ ), 136.9 (C-9), 139.0 (C-7), 140.9 (C-8), 161.1 (d, $\left.{ }^{1} J_{\mathrm{CF}}=240 \mathrm{~Hz}, \mathrm{C}-26\right), 165.0$ (C-19), 174.8 (OPv-7, OPv-8), 176.8 (OPv-3); (+)-LRESIMS m/z 708 (100) $[\mathrm{M}+\mathrm{H}]^{+}$; (+)-HRESIMS $m / z 708.4259[\mathrm{M}+\mathrm{H}]^{+}$(calcd for $\mathrm{C}_{42} \mathrm{H}_{59} \mathrm{FNO}_{7}, 708.4270$ ). *Interchangeable signals.

Compound 15: brown gum, (3.5 mg, 4\%); [ $[\alpha]_{\mathrm{D}}^{24}+54(c 0.1$, $\mathrm{MeOH}) ; \mathrm{UV}(\mathrm{MeOH})(\log \varepsilon) 208$ (4.92), 262 (4.08), 322 (3.49) $\mathrm{nm} ;{ }^{1} \mathrm{H}$ NMR $\left(800 \mathrm{MHz}, \mathrm{DMSO}-d_{6}\right) \delta_{\mathrm{H}} 0.55(3 \mathrm{H}, \mathrm{d}, J=5.6 \mathrm{~Hz}$, $\mathrm{H}-18), 1.13(9 \mathrm{H}, \mathrm{s}, \mathrm{OPv}-3), 1.23(9 \mathrm{H}, \mathrm{s}, \mathrm{OPv}-7), 1.20(3 \mathrm{H}$, overlap, H-20), 1.28 (1H, m, H-12b), 1.41 (1H, m, H-12a), 1.31 $(9 \mathrm{H}, \mathrm{s}, \mathrm{OPv}-8), 1.56(3 \mathrm{H}, \mathrm{s}, \mathrm{H}-17), 1.61(1 \mathrm{H}$, overlap H-2ß), 1.64 (3H, s, H-16), 1.95 (1H, m, H-13b), $2.01(1 \mathrm{H}, \mathrm{m}, \mathrm{H}-13 \mathrm{a}), 2.08$ $(1 \mathrm{H}$, overlap $\mathrm{H}-11), 2.11(1 \mathrm{H}$, overlap $\mathrm{H}-2 \alpha), 2.24(6 \mathrm{H}, \mathrm{s}, \mathrm{H}-25$, H-26), 2.46 (2H, t, $J=6.0 \mathrm{~Hz}, \mathrm{H}-23), 2.94(1 \mathrm{H}, \mathrm{m}, \mathrm{H}-1), 3.06$ $(1 \mathrm{H}, \mathrm{m}, \mathrm{H}-4), 3.24(1 \mathrm{H}, \mathrm{ddd}, J=13.0,6.0,5.6, \mathrm{H}-22 \mathrm{~b}), 3.29(1 \mathrm{H}$, ddd, $J=13.0,6.0,5.6, \mathrm{H}-22 \mathrm{a}), 5.10(1 \mathrm{H}, \mathrm{t}, J=7.3, \mathrm{H}-14), 5.20$ $(1 \mathrm{H}, \mathrm{dt}, J=11.04 .0 \mathrm{~Hz}, \mathrm{H}-3), 7.11(1 \mathrm{H}, \mathrm{s}, \mathrm{H}-5), 8.29(1 \mathrm{H}, \mathrm{t}, J=$ $5.6 \mathrm{~Hz}, \mathrm{NH}-21) ;{ }^{13} \mathrm{C}$ NMR $\left(200 \mathrm{MHz}, \mathrm{DMSO}-d_{6}\right) \delta_{\mathrm{C}} 17.5(\mathrm{C}-17)$, 18.4 (C-18), 22.8 (C-20), 25.5 (C-16), 25.8 (C-13), 26.8 (OPv-3), 26.9 (OPv-7, OPv-8), 28.1 (C-1), 31.1 (C-11), 31.3 (C-2), 37.2 (C-22), 37.4 (C-12), 38.3 (OPv-3), 38.4 (OPv-7), 38.6 (OPv-8), 45.1 (C-25, C-26), 45.5 (C-4), 57.8 (C-23), 68.8 (C-3), 124.1 (C14), 125.9 (C-5), 128.4 (C-6), 130.9 (C-15), 134.4 (C-10), 136.7 (C-9), 138.9 (C-7), 140.8 (C-8), 165.0 (C-19), 174.8 (OPv-7, OPv-8), 176.9 (OPv-3); (-)-LRESIMS m/z 671 (100) $[\mathrm{M}-\mathrm{H}]^{-}$;
(+)-HRESIMS $m / z 671.4625[\mathrm{M}+\mathrm{H}]^{+}$(calcd for $\mathrm{C}_{39} \mathrm{H}_{63} \mathrm{~N}_{2} \mathrm{O}_{7}$, 671.4625). "Interchangeable signals.

Compound 16: white amorphous powder, $(6 \mathrm{mg}, 7 \%)$; $[\alpha]_{\mathrm{D}}^{24}$ +30 (c 0.1, MeOH); UV (MeOH) (log $\varepsilon) 209$ (5.00), 241 (4.27) $\mathrm{nm} ;{ }^{1} \mathrm{H}$ NMR $\left(800 \mathrm{MHz}, \mathrm{DMSO}-d_{6}\right) \delta_{\mathrm{H}} 0.61(3 \mathrm{H}, \mathrm{d}, J=5.4 \mathrm{~Hz}$, H-18), 1.09 (9H, s, OPv-3), $1.18(1 \mathrm{H}, \mathrm{m}, \mathrm{H}-12 \mathrm{~b}), 1.22(3 \mathrm{H}$, overlap, H-20), 1.26 (9H, s, OPv-7), ${ }^{*} 1.32(9 \mathrm{H}, \mathrm{s}, \mathrm{OPv}-8),{ }^{*} 1.42$ (1H, m, H-12a), 1.57 (3H, s, H-17), $1.62(1 \mathrm{H}$, overlap, $\mathrm{H}-2 \beta)$, 1.64 (3H, s, H-16), 1.99 (2H, m, H-13), 2.09 (1H, overlap, H-2 $\alpha$ ), $2.12(1 \mathrm{H}$, overlap, H-11), $3.10(1 \mathrm{H}$, br t, $J=3.5 \mathrm{~Hz}, \mathrm{H}-4), 3.29$ $(1 \mathrm{H}$, br m, H-1) $5.11(1 \mathrm{H}, \mathrm{t}, J=7.0 \mathrm{~Hz}, \mathrm{H}-14), 5.25(1 \mathrm{H}, \mathrm{dt}, J=$ 11.0, 4.0 Hz, H-3), $7.56(1 \mathrm{H}, \mathrm{s}, \mathrm{H}-5) ;{ }^{13} \mathrm{C}$ NMR $(200 \mathrm{MHz}$, DMSO- $\left.d_{6}\right) \delta_{\mathrm{C}} 17.5$ (C-17), 18.4 (C-18), 22.7 (C-20), 25.5 (C-16), 25.8 (C-13), 26.7 (OPv-3), 26.9 (OPv-8), $27.0(\mathrm{OPv}-7),{ }^{*} 31.1(\mathrm{C}-$ 11), 36.2 (C-12), 38.3 (OPv-3), 38.5 (OPv-7), $38.6(\mathrm{OPv}-8),{ }^{*}$ 45.4 (C-4), 68.5 (C-3), 122.6 (C-6), 124.0 (C-14), 128.2 (C-5), 131.1 (C-15), 134.6 (C-10), 139.7 (C-7), 140.8 (C-9), 141.1 (C8), 165.4 (C-19), 174.8 (OPv-8), ${ }^{*} 175.2$ (OPv-7), ${ }^{*} 176.7$ (OPv-3); $(+)$-LRESIMS $m / z 623(100)[\mathrm{M}+\mathrm{Na}]^{+} ;(-)$-LRESIMS $m / z 599$ (100) $[\mathrm{M}-\mathrm{H}]^{-} ;(+)$-HRESIMS $m / z 623.3550[\mathrm{M}+\mathrm{Na}]^{+}$(calcd for $\left.\mathrm{C}_{35} \mathrm{H}_{52} \mathrm{O}_{8} \mathrm{Na}, 623.3554\right)$. *Interchangeable signals.

Compound 17: white amorphous powder, $(6.5 \mathrm{mg}, 9 \%)$; $[\alpha]_{\mathrm{D}}^{24}$ +28 (c 0.1, MeOH); UV (MeOH) (log $\varepsilon) 211$ (4.45), 240 (3.73), 306 (3.39) nm; ${ }^{1} \mathrm{H}$ NMR $\left(800 \mathrm{MHz}, \mathrm{DMSO}-d_{6}\right) \delta_{\mathrm{H}} 0.56(3 \mathrm{H}, \mathrm{d}, J$ $=6.9 \mathrm{~Hz}, \mathrm{H}-18), 1.10(9 \mathrm{H}, \mathrm{s}, \mathrm{OPv}-3), 1.19(3 \mathrm{H}$, overlap, H-20), $1.19(1 \mathrm{H}$, overlap, H-12a), $1.32(9 \mathrm{H}, \mathrm{OPv}-8), 1.42(1 \mathrm{H}$, overlap, H-12b), 1.56 (3H, s, H-17), 1.59 (1H, m, H-2ß), 1.64 (3H, s, H16), 1.98 (2H, m, H-13), $2.06(1 \mathrm{H}$, overlap, H-11), $2.10(1 \mathrm{H}, \mathrm{m}$, H-2 $\alpha), 2.99$ (1H, m, H-4), 3.03 (1H, br m, H-1), $5.11(1 \mathrm{H}, \mathrm{t}, J=$ $7.0 \mathrm{~Hz}, \mathrm{H}-14), 5.21(1 \mathrm{H}, \mathrm{dt}, J=11.0,4.0 \mathrm{~Hz}, \mathrm{H}-3), 7.42(1 \mathrm{H}, \mathrm{s}$, H-5), $11.19(1 \mathrm{H}$, br s. $7-\mathrm{OH}) ;{ }^{13} \mathrm{C}$ NMR $\left(200 \mathrm{MHz}\right.$, DMSO- $\left.d_{6}\right) \delta_{\mathrm{C}}$ 17.5 (C-17), 18.5 (C-18), 22.5 (C-20), 25.5 (C-16), 25.7 (C-13), 26.7 (OPv-3), 26.8 (OPv-8), 28.1 (C-1), 31.1 (C-2), 31.6 (C-11), $37.0(\mathrm{C}-12), 38.3(\mathrm{OPv}-3), 38.6(\mathrm{OPv}-8), 45.1(\mathrm{C}-4), 68.9$ (C-3), 111.5 (C-6), 124.1 (C-14), 126.5 (C-5), 126.9 (C-8), 131.0 (C15), 136.7 (C-10), 141.2 (C-9), 151.3 (C-7), 171.7 (C-19), 174.9 (OPv-8), 176.7 (OPv-3); (-)-LRESIMS $m / z 515(100)[\mathrm{M}-\mathrm{H}]^{-}$; $(+)$-HRESIMS $m / z 539.2982[\mathrm{M}+\mathrm{Na}]^{+}$(calcd for $\mathrm{C}_{30} \mathrm{H}_{44} \mathrm{O}_{7} \mathrm{Na}$, 539.2979).

Compound 18: white amorphous powder, (11.2 mg, 16\%); $[\boldsymbol{\alpha}] \mathrm{D}^{4}+52(c$ 0.1, MeOH); UV (MeOH) $(\log \varepsilon) 210$ (4.96), 240 (4.26), 296 (3.41) nm; ${ }^{1} \mathrm{H}$ NMR (800 MHz, DMSO- $\left.d_{6}\right) \delta_{\mathrm{H}} 0.39$ $(3 \mathrm{H}, \mathrm{d}, J=6.5 \mathrm{~Hz}, \mathrm{H}-18), 1.19(3 \mathrm{H}$, overlap, H-20), $1.25(9 \mathrm{H}, \mathrm{s}$, OPv-7), 1.32 (9H, s, OPv-8), $1.29(1 \mathrm{H}$, overlap, H-12b), $1.52(1 \mathrm{H}$, overlap, H-12a), 1.53 (1H, overlap, $\mathrm{H}-2 \beta), 1.58$ (3H, s, H-17), 1.66 (3H, s, H-16), 1.90 (1H, overlap, H-2 $\alpha$ ), 2.00 (2H, m, H-13), $2.17(1 \mathrm{H}, \mathrm{m}, \mathrm{H}-11), 2.81$ (1H, overlap, H-1), $2.89(1 \mathrm{H}$, br d, $J=$ $4.0 \mathrm{~Hz}, \mathrm{H}-4), 4.14(1 \mathrm{H}, \mathrm{m}, \mathrm{H}-3), 4.84(1 \mathrm{H}$, br s, OH-3), $5.14(1 \mathrm{H}$, t, $J=7.5 \mathrm{~Hz}, \mathrm{H}-14), 7.49$ (1H, s, H-5), 13.15 (1H, br s, OH-19); ${ }^{13} \mathrm{C}$ NMR (200 MHz, DMSO- $\left.d_{6}\right) \delta_{\mathrm{C}} 17.6$ (C-17), 18.9 (C-18), 23.1 (C-20), 25.6 (C-16), 25.7 (C-13), 26.9 (OPv-7), 27.0 (OPv-8), 28.9 (C-1), 30.0 (C-11), 34.1 (C-2), 38.4 (C-12), 38.5 (OPv-8), 38.7 (OPv-8), 48.1 (C-4), 64.1 (C-3), 122.3 (C-6), 124.7 (C-14), 129.0 (C-5), 130.5 (C-15), 136.2 (C-10), 139.9 (C-9), 140.6 (C7), 141.1 (C-8), 165.5 (C-19), 175.0 (OPv-7), 175.2 (OPv-8); (+)LRESIMS $m / z 539(100)[\mathrm{M}+\mathrm{Na}]^{+} ;(-)$-LRESIMS $m / z 515(100)$ $[\mathrm{M}-\mathrm{H}]^{-} ;(+)$-HRESIMS $\mathrm{m} / z$ 539.2982 $[\mathrm{M}+\mathrm{Na}]^{+}$(calcd for $\left.\mathrm{C}_{30} \mathrm{H}_{44} \mathrm{O}_{7} \mathrm{Na}, 539.2979\right)$

\section{Bioassays}

5.1 In vitro antimalarial image-based assay: The assay protocol has been published in detail previously. ${ }^{33}$ In brief, compounds were incubated in the presence of 2 or $3 \%$ parasitemia (3D7 or Dd2) at $0.3 \%$ hematocrit in a total assay volume of 50 
$\mu \mathrm{L}$, for $72 \mathrm{~h}$ at $37{ }^{\circ} \mathrm{C}$ and $5 \% \mathrm{CO}_{2}$ and $5 \% \mathrm{O}_{2}$, in poly-D-lysinecoated imaging plates. After incubation, the plates were stained with DAPI and incubated for a further $5 \mathrm{~h}$ at $\mathrm{rt}$ in the dark before imaging on the Opera HTS confocal imaging system. The digital images obtained were analyzed using Acapella spot detection software, whereby fluorescent spots which fulfilled the criteria established for a stained parasite were counted. The \% inhibition of parasite replication was calculated using $0.4 \%$ DMSO $(0 \%$ inhibition) and $5 \mu \mathrm{M}$ puromycin (100\% inhibition) control data. Artesunate and chloroquine were used as reference compound controls. $\mathrm{IC}_{50}$ values were obtained by plotting $\%$ inhibition against log dose using a graphing package and nonlinear regression with variable slope plot.

5.2 In vitro cytotoxicity assay: Compounds $(5 \mu \mathrm{L})$ were added to 384-well black/clear tissue culture treated assay plates containing 2000 adherent cells/well (HEK293) in an assay volume of $45 \mu \mathrm{L}$. The plates were incubated for $72 \mathrm{~h}$ at $37^{\circ} \mathrm{C}$ and $5 \% \mathrm{CO}_{2}$. After incubation, the supernatant was aspirated out of the wells and $40 \mu \mathrm{L}$ of $10 \%$ Alamar Blue was added per well. Plates were incubated for a further 5-6 $\mathrm{h}$ and measured for fluorescence at $535 \mathrm{~nm}$ excitation and $590 \mathrm{~nm}$ emission. The \% inhibition of cell growth was calculated using $0.4 \%$ DMSO (no inhibition) and $5 \mu \mathrm{M}$ puromycin (100\% inhibition) control data. $\mathrm{IC}_{50}$ values were obtained by plotting \% inhibition against log dose using a graphing package and nonlinear regression with a variable slope plot.

\section{Acknowledgments}

The authors acknowledge the National Health and Medical Research Council (NHMRC) for financial support (Grant APP1024314 to RAD) and thank the Australian Research Council (ARC) for support toward NMR and MS equipment (Grants LE0668477 and LE0237908) and financial support (Grant LP120200339 to RAD and LP120200557 to VMA). We acknowledge W. Loa (Griffith University) for acquiring the HRESIMS measurements. RK would like to thank Griffith University for PhD scholarships (GUPRS and GUIPRS).

\section{Supplementary Material}

Supplementary material that may be helpful in the review process should be prepared and provided as a separate electronic file. That file can then be transformed into PDF format and submitted along with the manuscript and graphic files to the appropriate editorial office.

\section{References and notes}

$\begin{array}{cc}\begin{array}{c}\text { 1. } \\ \text { 2. }\end{array} & \text { Kingston, D. G. I. J. Nat. Prod. 2011, 74, } 496 . \\ \text { 79, 629. } & \text { Lewman, D. J.; Cragg, G. M. J. Nat. Prod. 2016, } \\ \text { 3. } & \text { Lachance, H.; Wetzel, S.; Kumar, K.; Waldmann, } \\ \text { H. J. Med. Chem. 2012, 55, 5989. } \\ \text { 4. } & \text { Patridge, E.; Gareiss, P.; Kinch, M. S.; Hoyer, D. } \\ \text { Drug Discov. Today 2016, 21, 204. }\end{array}$

5. Schwarz, O.; Jakupovic, S.; Ambrosi, H.-D.; Haustedt, L. O.; Mang, C.; Müller-Kuhrt, L. J. Comb. Chem. 2007, 9, 1104.

Acta 2013, 1830, 3670.

6. Cragg, G. M.; Newman, D. J. Biochim. Biophys.

$7 . \quad$ Morrison, K. C.; Hergenrother, P. J. Nat. Prod.

Rep. 2014, 31, 6.

8. $\quad$ Barnes, E. C.; Kumar, R.; Davis, R. A. Nat. Prod Rep. 2016, 33, 372.

9. $\quad$ Pandey, R. C.; Toussaint, M. W.; Stroshane, R. M.; Kalita, C. C.; Aszalos, A. A.; Garretson, A. L.; Wei, T. T.; Byrne, K. M.; Geoghegan, R. F.; White, R. J. J. Antibiot. 1981, 34, 1389.
10. Abel, U.; Simon, W.; Eckard, P.; Hansske, F. G. Bioorg. Med. Chem. Lett. 2006, 16, 3292.

11. Sakai, R.; Higa, T.; Jefford, C. W.; Bernardinelli, G. J. Am. Chem. Soc. 1986, 108, 6404.

12. Peng, J.; Kudrimoti, S.; Prasanna, S.; Odde, S.; Doerksen, R. J.; Pennaka, H. K.; Choo, Y.-M.; Rao, K. V.; Tekwani, B. L.; Madgula, V.; Khan, S. I.; Wang, B.; Mayer, A. M. S.; Jacob, M. R.; Tu, L. C.; Gertsch, J.; Hamann, M. T. J. Med. Chem. 2010, 53, 61.

13. Tomishima, M.; Ohki, H.; Yamada, A.; Maki, K.; Ikeda, F. Bioorg. Med. Chem. Lett. 2008, 18, 1474.

14. Iwamoto, T.; Fujie, A.; Sakamoto, K.; Tsurumi, Y.; Shigematsu, N.; Yamashita, M.; Hashimoto, S.; Okuhara, M.; Kohsaka, M. J. Antibiot. 1994, 47, 1084.

15. Barnes, E. C.; Choomuenwai, V.; Andrews, K. T.; Quinn, R. J.; Davis, R. A. Org. Biomol. Chem. 2012, 10, 4015.

16. Choomuenwai, V.; Andrews, K. T.; Davis, R. A. Bioorg. Med. Chem. 2012, 20, 7167.

17. Kumar, R.; Sadowski, M. C.; Levrier, C.; Nelson, C. C.; Jones, A. J.; Holleran, J. P.; Avery, V. M.; Healy, P. C.; Davis, R. A. J. Nat. Prod. 2015, 78, 914.

18. Barnes, E. C.; Kavanagh, A. M.; Ramu, S.; Blaskovich, M. A.; Cooper, M. A.; Davis, R. A. Phytochemistry 2013, 93, 162.

19. Biva, I. J.; Ndi, C. P.; Griesser, H. J.; Semple, S. J. J. Ethnopharmacol. 2016, 182, 1.

20. Sansinenea, E.; Ortiz, A. Curr. Org. Synth. 2016, 13,556 .

21. Mon, H. H.; Christo, S. N.; Ndi, C. P.; Jasieniak, M.; Rickard, H.; Hayball, J. D.; Griesser, H. J.; Semple, S. J. J. Nat. Prod. 2015, 78, 3031 .

22. Lanigan, R. M.; Starkov, P.; Sheppard, T. D. J. Org. Chem. 2013, 78, 4512.

23. Dunetz, J. R.; Xiang, Y.; Baldwin, A.; Ringling,

J. Org. Lett. 2011, 13, 5048.

24. Valeur, E.; Bradley, M. Chem. Soc. Rev. 2009, 38,606

25. Montalbetti, C. A. G. N.; Falque, V. Tetrahedron 2005, 61, 10827.

26. Pattabiraman, V. R.; Bode, J. W. Nature 2011, $480,471$.

27. Dunetz, J. R.; Magano, J.; Weisenburger, G. A. Org. Process Res. Dev. 2016, 20, 140.

28. Itoh, T.; Taguchi, T.; Kimberley, M. R.; BookerMilburn, K. I.; Stephenson, G. R.; Ebizuka, Y.; Ichinose, K. Biochemistry 2007, 46, 8181 .

29. Schwartz, B. D.; Skinner-Adams, T. S.; Andrews, K. T.; Coster, M. J.; Edstein, M. D.; MacKenzie, D.; Charman, S. A.; Koltun, M.; Blundell, S.; Campbell, A.; Pouwer, R. H.; Quinn, R. J.; Beattie, K. D.; Healy, P. C.; Davis, R. A. Org. Biomol. Chem. 2015, 13,1558 .

30. Davis, R. A.; Duffy, S.; Fletcher, S.; Avery, V. M.; Quinn, R. J. J. Org. Chem. 2013, 78, 9608.

31. Cambridge Soft, ChemDraw ${ }^{\circledR}$ Ultra version 14.0.0.118. www.cambridgesoft.com

32. Lipinski, C. A.; Lombardo, F.; Dominy, B. W.; Feeney, P. J. Adv. Drug Deliv. Rev. 1997, 23, 3.

33. Duffy, S.; Avery, V. M. Am. J. Trop. Med. Hyg.

$\mathbf{2 0 1 2}, 86,84$ 